\title{
Supporting information \\ On the coupling of electron transfer to proton transfer at electrified interfaces
}

\author{
Aimin Ge ${ }^{1}$, Georg Kastlunger ${ }^{2}$, Jinhui Meng ${ }^{1}$, Per Lindgren ${ }^{2}$, Jia Song ${ }^{1}$, Qiliang Liu ${ }^{1}$, \\ Alexander Zaslavsky ${ }^{2}$, Tianquan Lian ${ }^{1}$, and Andrew A. Peterson ${ }^{2}$ \\ ${ }^{1}$ Department of Chemistry, Emory University, Atlanta, Georgia 30322, USA \\ ${ }^{2}$ School of Engineering, Brown University, Providence, Rhode Island, 02912, USA
}

\section{Contents}

S1 Extended methods description $\quad$ S1

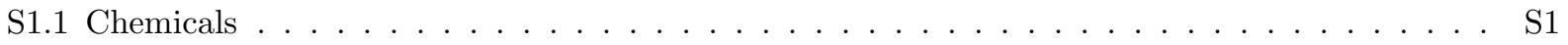

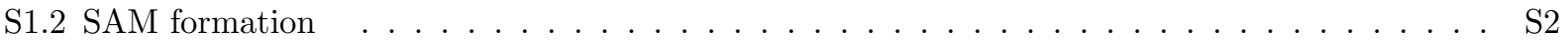

S1.3 Electrochemical SFG spectroscopy . . . . . . . . . . . . . . . . . . S2

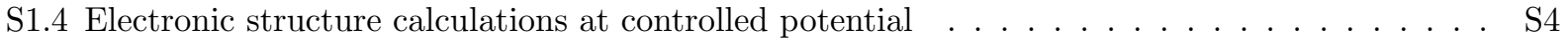

S1.5 Calculation of $\mathrm{p} K_{\mathrm{a}}$ in constant-charge and constant-potential simulations . . . . . . . . S S4

S1.6 Shape of the jellium counter charge . . . . . . . . . . . . . . . . . . . . S6

S2 Extended results analysis $\quad$ S6

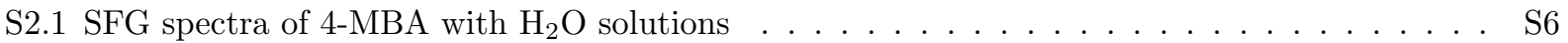

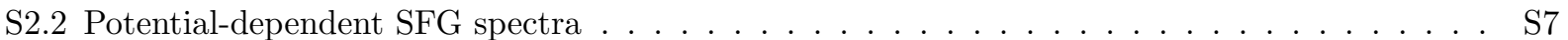

$\mathrm{S} 2.3 \mathrm{pH}$-dependent frequency shifts $\ldots \ldots \ldots \ldots \ldots \ldots \ldots \ldots$. . . . . . . . . . . . .

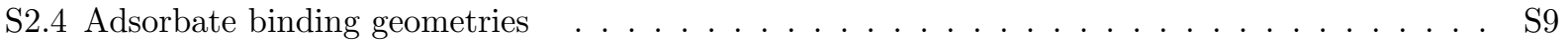

S2.5 Simulated IR spectra . . . . . . . . . . . . . . . . . . . . . . S10

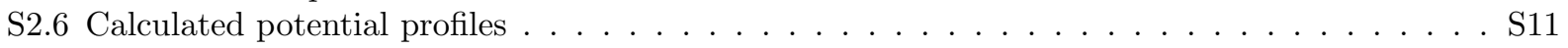

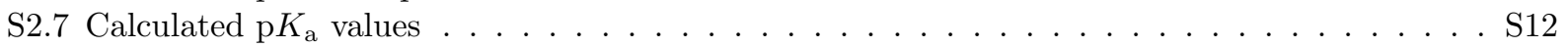

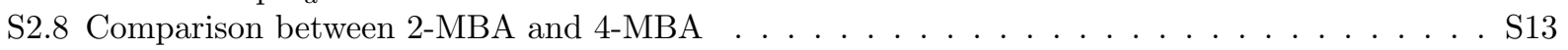

S3 Gauss's law model of charge-potential relation $\quad$ S14

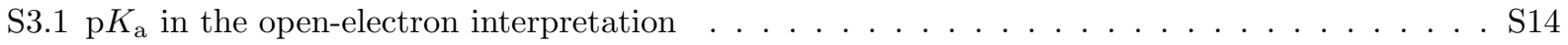

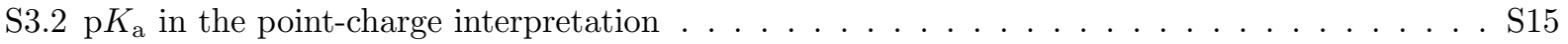

S3.3 Gauss's law equivalence . . . . . . . . . . . . . . . . . . . . . . S15

\section{S1. Extended methods description}

\section{S1.1. Chemicals}

4-mercaptobenzoic acid (99\%), $\mathrm{NaClO}_{4}$ (ACS reagent, $\geq 98 \%$ ) and $\mathrm{Na}_{3} \mathrm{PO}_{4}$ were purchased from SigmaAldrich. $\mathrm{H}_{3} \mathrm{PO}_{4}$ (ortho-phosphoric acid, 85\%) was received from Fisher Scientific. $\mathrm{Na}_{2} \mathrm{HPO}_{4}$ and $\mathrm{NaH}_{2} \mathrm{PO}_{4}$ were purchased from Macron Chemicals. Ultrapure $\mathrm{H}_{2} \mathrm{O}$ (MilliQ, $18.2 \mathrm{M} \Omega \cdot \mathrm{cm}$ ) or $\mathrm{D}_{2} \mathrm{O}(99.9$ atom \% D, 
Aldrich) were used to prepare solutions of $0.1 \mathrm{M} \mathrm{H}_{3} \mathrm{PO}_{4}, \mathrm{NaH}_{2} \mathrm{PO}_{4}, \mathrm{Na}_{2} \mathrm{HPO}_{4}$, and $\mathrm{Na}_{3} \mathrm{PO}_{4}$. These solutions were used to make buffer solutions in $\mathrm{H}_{2} \mathrm{O}$ or $\mathrm{D}_{2} \mathrm{O}$ with different $\mathrm{pH}$ values.

\section{S1.2. SAM formation}

Au thin films evaporated on a glass microscope slide (Substrata Thin Film Solutions) were used as substrates for 4-MBA self-assembled monolayer (SAM) formation. The thickness of the gold layer is $100 \mathrm{~nm}$. A titanium adhesion layer was used to bind the gold to the glass surface. The Au slides $(25 \mathrm{~mm} \times 10 \mathrm{~mm})$ were immersed in a piranha solution $\left(\mathrm{H}_{2} \mathrm{SO}_{4}: \mathrm{H}_{2} \mathrm{O}_{2}=3: 1\right)$ for $10 \mathrm{~min}$ and then rinsed by pure water. After that, the gold slides were washed by ethanol and dried by nitrogen. The Au slides were immersed in a 5-mM 4-MBA solution in ethanol for 72 hours at $25^{\circ} \mathrm{C}$. After SAM formation, the Au slides were washed by ethanol and dried by nitrogen stream.

Ultrapure $\mathrm{H}_{2} \mathrm{O}$ or $\mathrm{D}_{2} \mathrm{O}$ was used to prepare a phosphate buffer solution. The $\mathrm{pH}$ of the buffer solutions was measured by a $\mathrm{pH}$ meter (Acuumet, AB15). To facilitate comparisons between measurements at different $\mathrm{pH}$ levels, the ionic strength was held constant in all buffers. This was set to $0.2 \mathrm{M}$ by adding $\mathrm{NaClO}_{4}$. The measured $\mathrm{pH}^{*}$ in $\mathrm{D}_{2} \mathrm{O}$ buffer has been converted to $\mathrm{pH}$ in $\mathrm{H}_{2} \mathrm{O}$ buffer by $\mathrm{pH}=0.929 \mathrm{pH}^{*}+0.42$. [1] The buffer solutions were de-aerated by nitrogen gas (>99.999\%, Nexair) for 15 min before use.

\section{S1.3. Electrochemical SFG spectroscopy}

The electrochemical cell setup is shown in Figure S-1 and is similar to the one used in our previous works [2,3]. The Au slide was directly attached on a glass dish bottom by epoxy resin and used as a working electrode. Then, conducting copper tape was connected to the surface of the Au slide. The copper tape was isolated from the electrolyte by epoxy resin. For the SFG measurements in $\mathrm{H}_{2} \mathrm{O}$ buffer, a 2-mm thick $\mathrm{CaF}_{2}$ plate was pressed on the surface of the $\mathrm{Au}$ slide to reduce the infrared absorption from the thin electrolyte layer. In the case of $\mathrm{D}_{2} \mathrm{O}$ buffer, a $25-\mu$ m-thick Teflon spacer was added between the $\mathrm{CaF}_{2}$ window and Au surface. Between SFG measurements with different solutions, the electrochemical cell was rinsed with ultrapure water and the solution to be used next. Pt wire and $\mathrm{Ag} / \mathrm{AgCl}(1 \mathrm{M} \mathrm{KCl})$ electrode were used as counter and reference electrodes, respectively. A potentiostat ( $\mathrm{CH}$ Instruments) was used to control the potentials (from $0.1 \mathrm{~V}$ to $-0.5 \mathrm{~V}$ ) during SFG measurements.

As a second-order nonlinear spectroscopic technique [4,5], SFG provides an intrinsically surface-specific in situ probe of adsorbates on planar electrodes [4,6-8]. 4-MBA was chosen because the population of carboxylate groups can be conveniently tracked by SFG and the pH-insensitive ring mode can be used as an internal reference of relative surface density [9-11]. A high-repetition-rate femtosecond laser system (Carbide, $200 \mathrm{kHz}$, Light Conversion) was used for the broadband SFG measurement. A built-in pulse picker is used for pulse-on-demand control. The Carbide laser generates $229 \mathrm{fs}$ pulses at $1030 \mathrm{~nm}$ with a pulse energy of $200 \mu \mathrm{J} .90 \%$ of the Carbide output (compressed) is used to pump a high power collinear optical parametric amplifier (Orpheus-HP, Light Conversion) to produce tunable IR pulses. The other 10\% of the Carbide output (uncompressed) is used to pump a second harmonic bandwidth compressor (SHBC, Light Conversion) to generate narrow-band $515 \mathrm{~nm}$ pulse with a bandwidth of $6 \mathrm{~cm}^{-1}$. In this work, a repetition rate of $10 \mathrm{kHz}$ was used to avoid sample damage. The pulse energies and beam waists (in parenthesis) of IR and visible beams on the sample surface are $\sim 2 \mu \mathrm{J}(\sim 500 \mu \mathrm{m})$ and $\sim 4 \mu \mathrm{J}(\sim 650 \mu \mathrm{m})$, respectively. The angles of incidence are $40^{\circ}$ for IR and $65^{\circ}$ for visible beams. All SFG measurements were carried out under $p p p$ polarization combination ( $p$-SFG, $p$-visible, $p$-IR). SFG signal was dispersed by a spectrograph (Shamrock, Andor) and detected by an electron-multiplied charge coupled device (Newton EMCCD, Andor).

A home-made electrochemical cell was used for the SFG measurements under bias (Figure S-1) [2]. Two SFG spectra measured with IR beams centered at $6200 \mathrm{~nm}$ and $7200 \mathrm{~nm}$ were combined to produce SFG spectra that cover a broad IR frequency range $\left(1350 \sim 1800 \mathrm{~cm}^{-1}\right)$. Only the normalized SFG spectra are shown below in which the variation of incident IR beam intensity as a function of wavelength has been removed by normalizing the SFG spectra from the 4-MBA samples by those from the bare gold/neutral pH solution interface. The latter were obtained by reductively removing the 4-MBA SAM from the gold surface at negative potentials. The acquisition time for one spectrum is typically $1 \mathrm{~min}$. All measurements were 


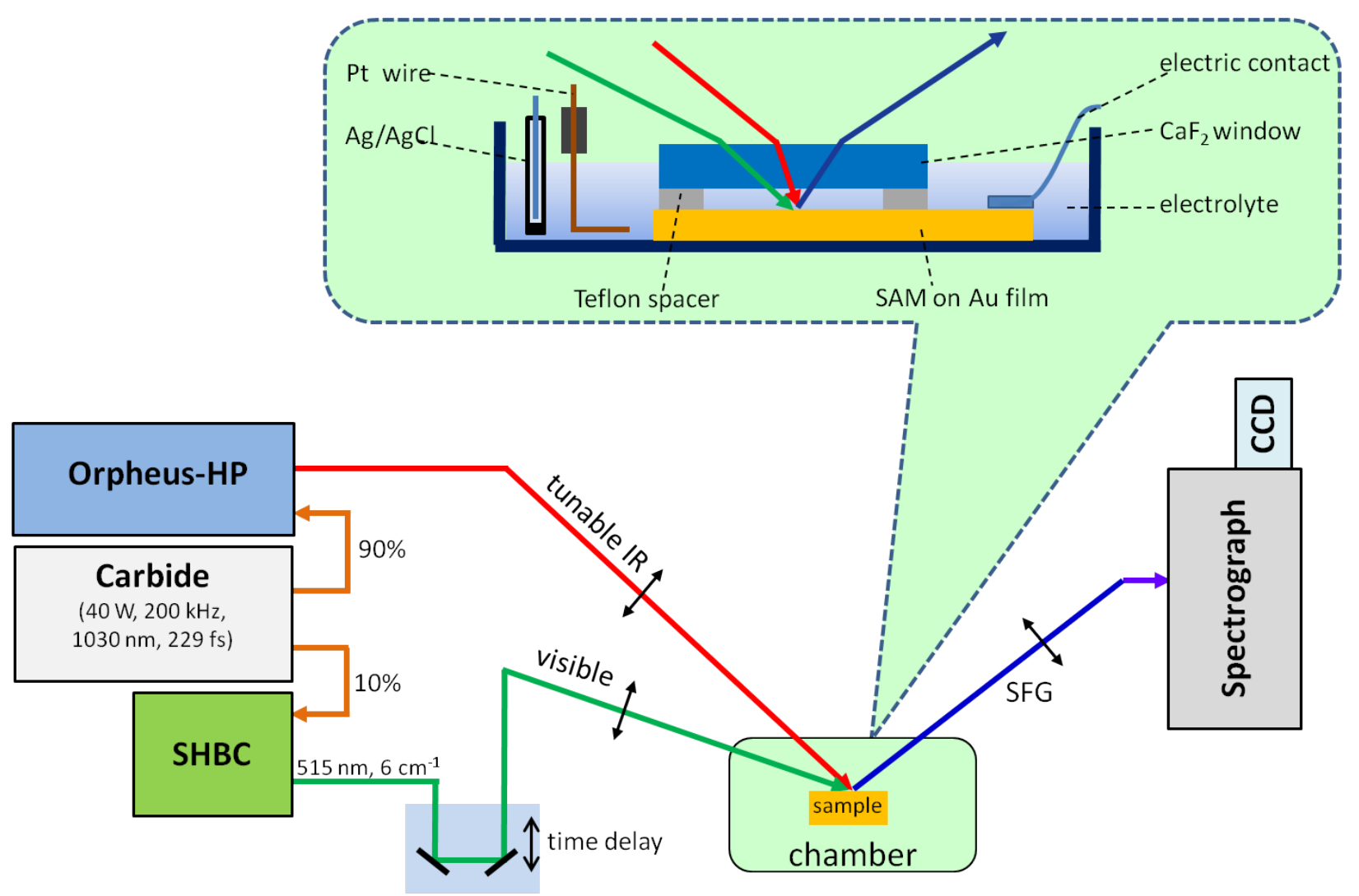

Figure S-1: Electrochemical SFG setup used in this work. 
performed at room temperature. No photo-induced degradation of 4-MBA monolayers was observed during the SFG experiment. Usually, it took 10 15 hours to collect potential-dependent SFG spectra of one sample in solution with varying $\mathrm{pH}$ values.

The SFG spectra were fit using the following expression [5,12],

$$
I_{\mathrm{SFG}} \propto\left|A_{\mathrm{NR}} e^{i \phi_{\mathrm{NR}}}+\sum_{n} \frac{A_{n}}{\omega_{\mathrm{IR}}-\omega_{n}+i \Gamma_{n}}\right|^{2}
$$

where $A_{\mathrm{NR}}$ and $\phi_{\mathrm{NR}}$ are the magnitude and phase of the non-resonant SFG response from the gold surface, respectively. $A_{n}, \omega_{n}$, and $\Gamma_{n}$ are the amplitude, peak frequency, and damping constant of the $n^{\text {th }}$ vibrational mode of the 4-MBA molecules. $A_{n}$ is proportional to the number density of the molecules on the sample surface.

\section{S1.4. Electronic structure calculations at controlled potential}

To perform controlled-potential electronic structure calculations, the solvated jellium (SJ) method was applied. [13] This method controls the electrode potential (that is, the work function) constant by adding or subtracting electrons (or fractions thereof) to the unit cell. The SJ method is implemented in the GPAW [14,15] electronic structure code. In this scheme, the effective potential cavity scheme of Held and Walter [16] as implemented in GPAW was used for the simulation of the implicit solvent. The parameters for the implicit solvent have been chosen according to ref. [13], being $u_{0}=0.180 \mathrm{eV}$ and $\gamma=0.0184 \mathrm{~Pa} \cdot \mathrm{m}$. The water bulk solvent dielectric constant was chosen as 78.36, and a temperature of $298.15 \mathrm{~K}$ was used in the Boltzmann distribution for the definition of the cavity. In contrast to previous studies where we applied the SJ method, $[13,17,18]$ the counter charge was chosen to be shaped in the same way as the shape function defining the local dielectric constant, as described in detail in a subsequent section of this document. Such an approach allows a qualitative simulation of the penetration of the monolayer by the electrolyte.

A key objective of this study is to determine the number of electrons $n$ that are required to hold the potential constant when the acid dissociation reaction occurs, as defined in equation (S.3). This quantity comes directly from these grand-canonical simulations: we simply compare the total number of electrons between simulations of the acid and the anion, for any given potential and geometry.

The electronic structure calculations have been performed in density functional theory (DFT) in the GPAW code. [14,15] A grid basis with an average grid spacing of $0.17 \AA$ has been applied, and the Bayesian error estimation XC-functional with dispersion correction (BEEF-vdW) [19] as implemented in libvdwxc [20] was used. The electrode surface was simulated as $\mathrm{Au}(111)$, where an equilibrium lattice constant of 4.26 Å was determined. The Au slab was created consisting of three layers perpendicular to the electrode surface, where the lowest layer was constrained at the determined lattice constant. The applied number of $k$-points along a given lattice vector direction was 10, 6, 4 for 1, 2, 3 gold layers, respectively. The direction perpendicular to the surface was sampled by a single $k$-point, and a dipole correction has been applied. A Fermi temperature of $0.1 \mathrm{eV}$ was applied. All geometries have been relaxed until a convergence criterion of $0.05 \mathrm{eV} / \AA$ for the atomic forces was reached.

Potential energies have been converted to free energies by standard methods,

$$
G=E+\Delta(\mathrm{ZPE})+\int_{0}^{T} C_{P} d T-T \Delta S
$$

where $E$ is the potential energy extrapolated to $0 \mathrm{~K}$. Zero-point energies (ZPE), heat capacities $\left(C_{p}\right)$, and entropy $(S)$ have been calculated from normal mode analyses in the harmonic limit at $298 \mathrm{~K}$.

\section{S1.5. Calculation of $\mathrm{p} K_{\mathrm{a}}$ in constant-charge and constant-potential simulations}

Constant-charge conditions (non-electrochemical). We start by considering the calculation of $\mathrm{p} K_{\mathrm{a}}$ on a $\mathrm{Au}$ surface which is not connected to an external circuit. By definition, the $\mathrm{p} K_{\mathrm{a}}$ is proportional to the 
standard free energy of reaction $\left(\Delta G_{\mathrm{rxn}}^{\circ}\right)$ for the dissociation of the acid-base pair $\left(\mathrm{HA} / \mathrm{A}^{-}\right)$, following the relationship

$$
\mathrm{p} K_{\mathrm{a}}=-\log K_{\mathrm{a}}=\frac{\Delta G_{\mathrm{rxn}}^{\circ}}{\ln 10 \cdot k_{\mathrm{B}} T}
$$

with

$$
\Delta G_{\mathrm{rxn}}^{\circ}=\mu^{\circ}\left[\mathrm{A}^{-}\right]+\mu^{\circ}\left[\mathrm{H}^{+}\right]-\mu^{\circ}[\mathrm{HA}]
$$

where $K_{\mathrm{a}}, k_{\mathrm{B}}$, and $T$ represent the dissociation constant, Boltzmann's constant and temperature, respectively. The chemical potential of the protonated $\left(\mu^{\circ}[\mathrm{HA}]\right)$ and deprotonated $\left(\mu^{\circ}\left[\mathrm{A}^{-}\right]\right)$acid can be readily calculated in the harmonic approximation from the vibrational free energies of the respective species.

Accounting for the chemical potential of a solvated proton is more complicated; however, we are able to use a Born-Haber-type approach [21] on the basis of the standard hydrogen equilibrium at $0 \mathrm{~V}_{\mathrm{SHE}}$ and $\mathrm{pH} 0$ (even though this reaction is not electrochemical - this is merely a convenient reference). Since the proton reservoir is that of the bulk solvent; this is a similar framework to the well-known computational hydrogen electrode [22], but decoupling the proton and electron energy as we discussed at length in a previous study [17]. Doing so $\mu^{\circ}\left[\mathrm{H}^{+}\right]$can be calculated as

$$
\mu^{\circ}\left[\mathrm{H}^{+}\right]=\frac{1}{2} \mu^{\circ}\left[\mathrm{H}_{2}\right]-\mu^{0 \mathrm{~V}}\left[\mathrm{e}^{-}\right]
$$

where $\mu^{\circ}\left[\mathrm{e}^{-}\right]$and $\mu^{0 \mathrm{~V}}\left[\mathrm{H}_{2}\right]$ represent the chemical potential of the electron at $0 \mathrm{~V}_{\mathrm{SHE}}$ and of $\mathrm{H}_{2}$ in the gas phase at standard conditions, respectively. We refer to the above as a "decoupled computational electrode". $\mu^{\circ}\left[\mathrm{H}_{2}\right]$ was calculated in the ideal-gas limit, and the absolute value of the standard hydrogen electrode was taken based on Trasatti's value of $\mu^{\circ}\left[\mathrm{e}^{-}\right]=-4.44 \mathrm{~V}[23]$. The $\mathrm{p} K_{\mathrm{a}}$ can now be calculated as

$$
\mathrm{p} K_{\mathrm{a}}=\frac{\mu^{\circ}\left[\mathrm{A}^{-}\right]+\frac{1}{2} \mu^{\circ}\left[\mathrm{H}_{2}\right]-\mu^{0 \mathrm{~V}}\left[\mathrm{e}^{-}\right]-\mu^{\circ}[\mathrm{HA}]}{\ln 10 \cdot k_{\mathrm{B}} T}
$$

Constant-potential conditions (electrochemical). If the surface is now connected to a potentiostat, one has to account for the constant-potential condition. [24] Since charge and potential (or more precisely, number of electrons and electrons' chemical potential) are conjugate thermodynamic variables, if we fix the potential then the number of electrons must be a free variable. In this case, our chemical reaction is written as:

$$
* \mathrm{AH}+n \mathrm{e}^{-} \stackrel{\phi}{\longrightarrow} * \mathrm{~A}^{(1+n)-}+\mathrm{H}^{+}
$$

We emphasize that the superscript ${ }^{(1+n)}$ - accounts for the difference in charge between $* \mathrm{AH}$ and $* \mathrm{~A}$, not the specific charge on $*$ A. Accounting for a constant electrode potential in practicably sized unit cells demands that one changes the simulated ensemble into a semi-grand canonical one, where electrons are allowed to flow from and into an electron reservoir to equilibrate the electrode potential. Such a treatment is still rather novel and has been developed following different strategies [25-29]. We have recently published an efficient method to perform constant-potential reaction simulation, known as the solvated jellium method [13]. We describe this approach in more detail in Section S1.4. In this scheme, electrons can be introduced or extracted from the periodic unit cell, while the net charge in the cell is unchanged. This is achieved by compensating the extra electrons in the atomic system with a spread-out counter charge, which is located in an implicit solvent on the reactive side of the slab. We emphasize that a slightly different approach in defining the counter charge has been chosen in this study. The counter charge shape has been defined to follow the shape of the implicit solvent cavity (or shape function). With this scheme, penetration of the SAM with counterions can be approximated.

Using a grand-canonical scheme such as the SJ method, we can directly infer the value of $n$ based on the difference in the number of electrons between the deprotonated $\left(* \mathrm{~A}^{-}\right)$and the protonated $(* \mathrm{AH})$ simulations. 
As discussed in Section $\mathrm{S} 3.1$, the $\mathrm{p} K_{\mathrm{a}}$ is now a function of potential, since the $\Delta G_{\mathrm{rxn}}^{\circ}$ is written as a function of potential:

$$
\Delta G_{\text {rxn }}^{\circ}(\phi)=\mu^{\phi}\left[* \mathrm{~A}^{(1+n)-}\right]+\mu^{\circ}\left[\mathrm{H}^{+}\right]-\mu^{\phi}[* \mathrm{AH}]-n \mu^{\phi}\left[\mathrm{e}^{-}\right]
$$

Again using a decoupled computational electrode approach [17,21], we substitute in equation (S.2):

$$
\Delta G_{\mathrm{rxn}}^{\circ}(\phi)=\mu^{\phi}\left[* \mathrm{~A}^{(1+n)-}\right]-\mu^{\phi}[* \mathrm{AH}]-(1+n) \mu^{\phi}\left[\mathrm{e}^{-}\right]+\frac{1}{2} \mu^{\circ}\left[\mathrm{H}_{2}\right]-e \phi
$$

where we have used the substitution that $\mu^{0 \mathrm{~V}}\left[\mathrm{e}^{-}\right]-\mu^{\phi}\left[\mathrm{e}^{-}\right]=e \phi$ when $\phi$ is the potential expressed versus $\mathrm{SHE}$, and $e$ is the charge on an electron (or Faraday constant, in molar units). Conveniently, the term that accounts for the difference in electrons between the two simulations, $(1+n) \mu^{\phi}\left[\mathrm{e}^{-}\right]$, is automatically included when using the SJ calculator [13] in GPAW as the grand-potential energy is returned by default.

\section{S1.6. Shape of the jellium counter charge}

In its original implementation, the solvated-jellium method [13] defines the jellium background charge as a region on top of the atomic system located inside the implicit solvent. The shape in this original description was chosen as a cuboid; that is, a "slab" that is periodic in the $x$ and $y$ directions and has a lower and upper limit in the $z$ direction, which is orthogonal to the electrode surface. This leads to a planar interface between the background charge and the atomic system. In order to allow the countercharge, mimicking the counterions in the double-layer, to penetrate the SAM, this definition has been generalized. In the new definition the charge density created by the counter charge is defined as

$$
\rho_{\mathrm{bg}}(\mathbf{r})= \begin{cases}\frac{q}{V} f(\mathbf{r}), & \text { for } r_{z}<z_{\mathrm{ul}} \\ 0, & \text { for } r_{z} \geq z_{\mathrm{ul}}\end{cases}
$$

where $q$ is the total charge included in the background charge region, $f$ corresponds to an arbitrary function in space, $z_{\mathrm{ul}}$ is the chosen value for the upper limit of the jellium background region and $V$ is given by

$$
V=\int^{z_{\mathrm{ul}}} f(\mathbf{r}) d \mathbf{r}
$$

The reason why no lower limit for the integration is given is that the desired function should generally approach zero close to the explicit system in order to avoid artifacts. An intuitive choice for $f(\mathbf{r})$ made in the current work is to follow the dielectric shape function $\varepsilon(\mathbf{r})$, since it satisfies the given demand and additionally describes the solvent- (or electrolyte-) accessible surface. We note that in the SJ method the backside of the electrode is not solvated, which is why $\varepsilon(\mathbf{r})$ will only locate the counter charge on the reactive side of the slab. An example of the jellium background charge shape for both the untilted and tilted $\mathrm{p}(2 \times 2)$ 4-MBA SAM are given in Figure S-2. While for the untilted structure, the jellium counter charge penetrates the SAM considerably, the penetration of the tilted SAM is nearly negligible.

\section{S2. Extended results analysis}

\section{S2.1. SFG spectra of 4-MBA with $\mathrm{H}_{2} \mathrm{O}$ solutions}

Figure S-3 shows the SFG spectra for 4-MBA with $\mathrm{H}_{2} \mathrm{O}$ solutions with varying $\mathrm{pH}$ values. The peak intensity and frequency of the $1400 \mathrm{~cm}^{-1}$ band show similar trends as those with $\mathrm{D}_{2} \mathrm{O}$ solutions. The $\nu_{\text {ring }}$ band is strongly influenced by the wing of the $\mathrm{H}_{2} \mathrm{O}$ bend band at higher than $1600 \mathrm{~cm}^{-1}$. At high $\mathrm{pH}$, a weak band around $1485 \mathrm{~cm}^{-1}$ can be observed (Figures S-4a and S-4b), which can be tentatively attributed to the asymmetric stretching mode of the $\mathrm{COO}^{-}$group as revealed by DFT calculations (Section S2.5). Similar weak peaks were observed for the spectra with $\mathrm{D}_{2} \mathrm{O}$ solutions. This assignment is supported by the observation that similar to the $1400 \mathrm{~cm}^{-1}$ band, the intensity of the $1485 \mathrm{~cm}^{-1}$ band also decreases as the 

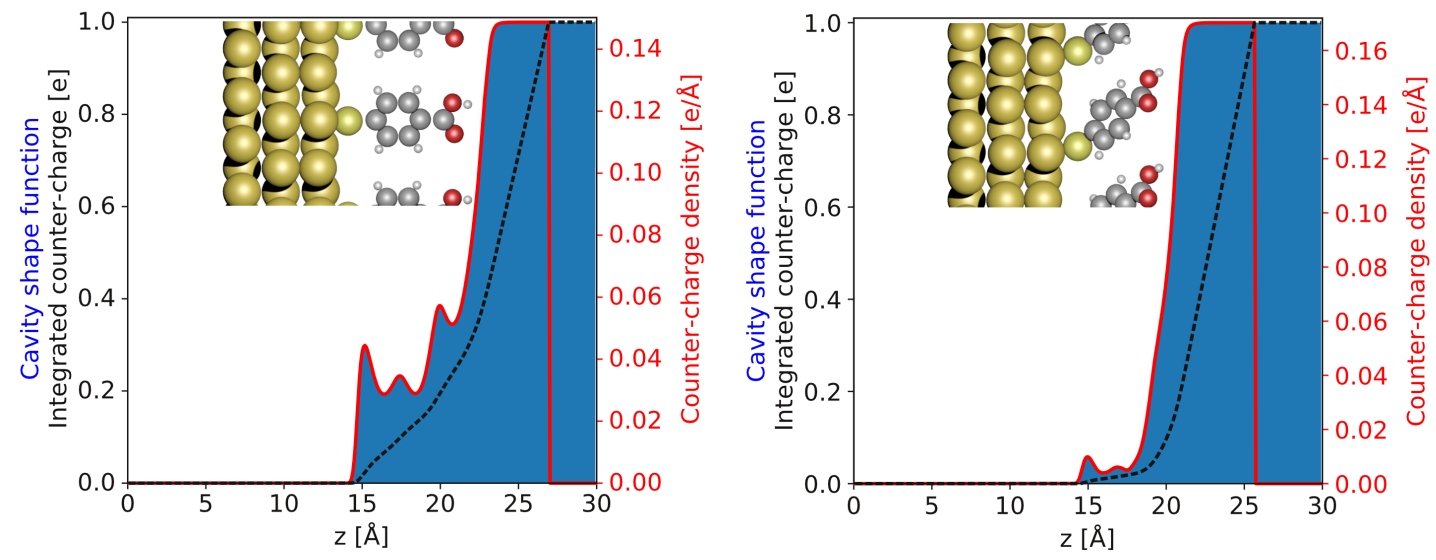

Figure S-2: Counter-charge distribution for the example of $\mathrm{p}(2 \times 2)$ non-tilted (left panel) and tilted (right) 4-MBA SAM at an applied charge of -1 electron.

$\mathrm{pH}$ decreases, and in the potential window of $0.1 \mathrm{~V}$ to $-0.5 \mathrm{~V}$, there is no apparent change of the amplitude ratio between the $1400 \mathrm{~cm}^{-1}$ band and $1485 \mathrm{~cm}^{-1}$ band (Figure S-4). However, the signal amplitude of the $1485 \mathrm{~cm}-1$ band is small, which hinders accurate determination of its $\mathrm{pH}$ and potential dependent peak position and intensity.

\section{S2.2. Potential-dependent SFG spectra}

Figure S-5(a) shows an example of the potential-dependent SFG spectra at $\mathrm{pH} 6$. When the applied potential changes from $0.1 \mathrm{~V}$ to $-0.5 \mathrm{~V}$ (vs. $\mathrm{Ag} / \mathrm{AgCl} 1 \mathrm{M} \mathrm{KCl}$ ), the intensity of the $\nu_{\mathrm{COO}^{-}}^{\mathrm{ss}}$ band decreases and the intensity of the $\nu_{\mathrm{COOD}}$ band increases, indicating a potential-induced change of the acid-base equilibrium. All potential-dependent SFG spectra are fitted to obtain the amplitude ratios of $A_{\mathrm{COO}^{-}}^{\mathrm{ss}} / A_{\text {ring }}$ and $A_{\mathrm{COOD}} / A_{\text {ring }}$ as a function of applied potential and pH. Shown in Figure S-5(b) is a plot of these ratios as a function of potential, also at $\mathrm{pH} 6$.

\section{S2.3. $\quad$ pH-dependent frequency shifts}

The SFG spectra were fit by equation (S.1) to obtain the frequency and amplitude of the modes. Figure 1a of the main article shows a pH-dependent frequency shift of the $\nu_{\mathrm{COO}}^{\mathrm{ss}}$ band. As shown in Figure 1c of the main article, the fitted frequency of $\nu_{\mathrm{COO}}^{\mathrm{ss}}$ shifts from $1385 \mathrm{~cm}^{-1}$ at $\mathrm{pH} 5$ to $1410 \mathrm{~cm}^{-1}$ at $\mathrm{pH}>10$. Similar behavior was also observed for experiments with $\mathrm{H}_{2} \mathrm{O}$ buffer (Figure S-3). The origin of this shift is unclear. As visible in Figure S-5, at a given $\mathrm{pH}$, the frequency of this mode shows negligible dependence on applied potential, suggesting that it cannot be attributed to an electrochemical Stark effect. [2,3,30,31] A similar pHdependent shift has been observed in related 2-MBA SAMs on Ag electrodes and has been attributed to the interaction between the $\mathrm{COO}^{-}$groups and the electrode surface. [32] As reported previously, the use of parasubstituted 4-MBA with saturated surface density on Au significantly reduces the direct interaction between the carboxylate species and the Au surface in comparison with 2-MBA and 3-MBA. [33] Furthermore, the fit of the SFG titration curve shown in Figure 1(b) of the main article suggests the existence of a significant $\mathrm{pH}$-dependent interaction energy between the $\mathrm{COO}^{-}$groups. It is possible that such interaction gives rise to the pH-dependent frequency shift directly or indirectly, through the change of adsorbate geometry.

Our electronic structure calculations can help us understand both the measured SFG spectra and to provide insights into the binding structure. Based on the calculations (Section S2.4) of the binding energy 


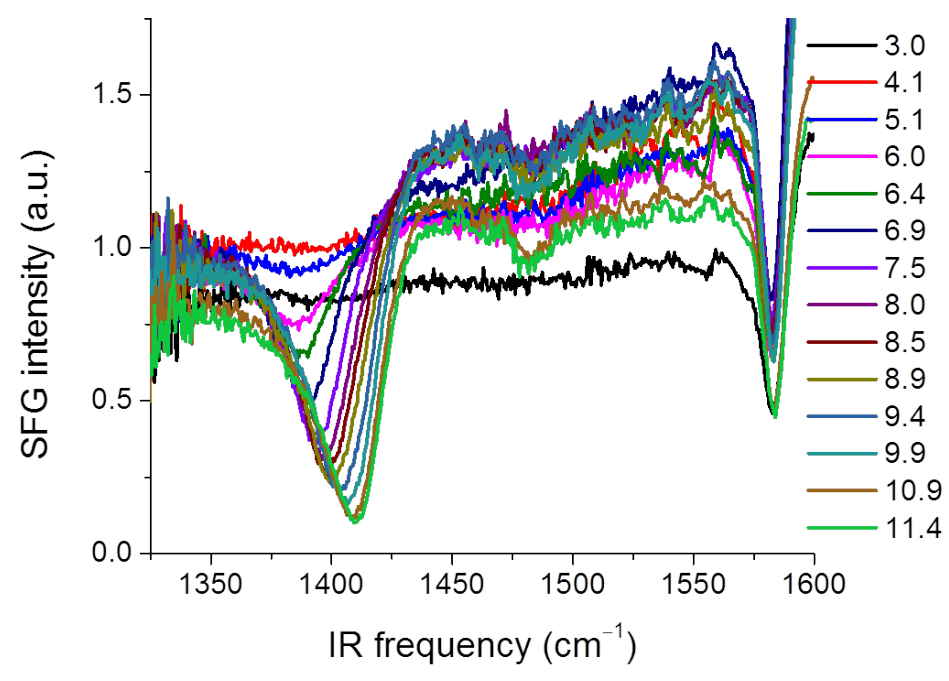

Figure S-3: SFG spectra of 4-MBA SAM in $\mathrm{H}_{2} \mathrm{O}$ buffer solutions with varying $\mathrm{pH}$ values without applying bias. The $\mathrm{pH}$ values are indicated in the figure.
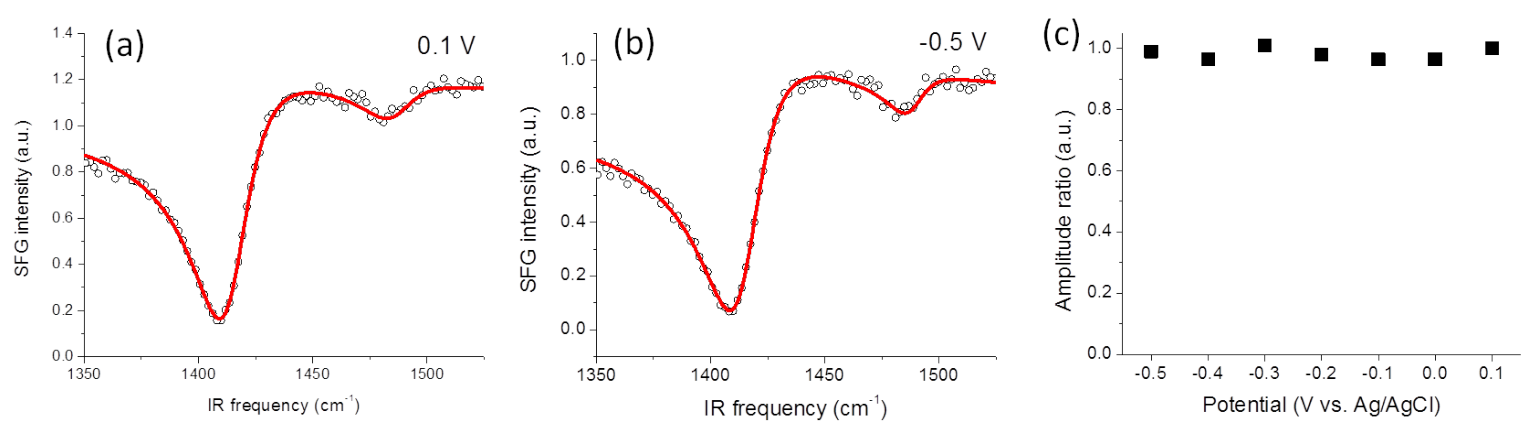

Figure S-4: SFG spectra of 4-MBA at (a) $0.1 \mathrm{~V}$ and (b) $-0.5 \mathrm{~V}$ with $\mathrm{pH} 11.4 \mathrm{H}_{2} \mathrm{O}$ solution. The open circles are experimental data. Solid curves are fits according to Equation S.1. (c) Normalized amplitude ratio between the $1400 \mathrm{~cm}^{-1}$ and $1485 \mathrm{~cm}^{-1}$ modes as a function of potential. 

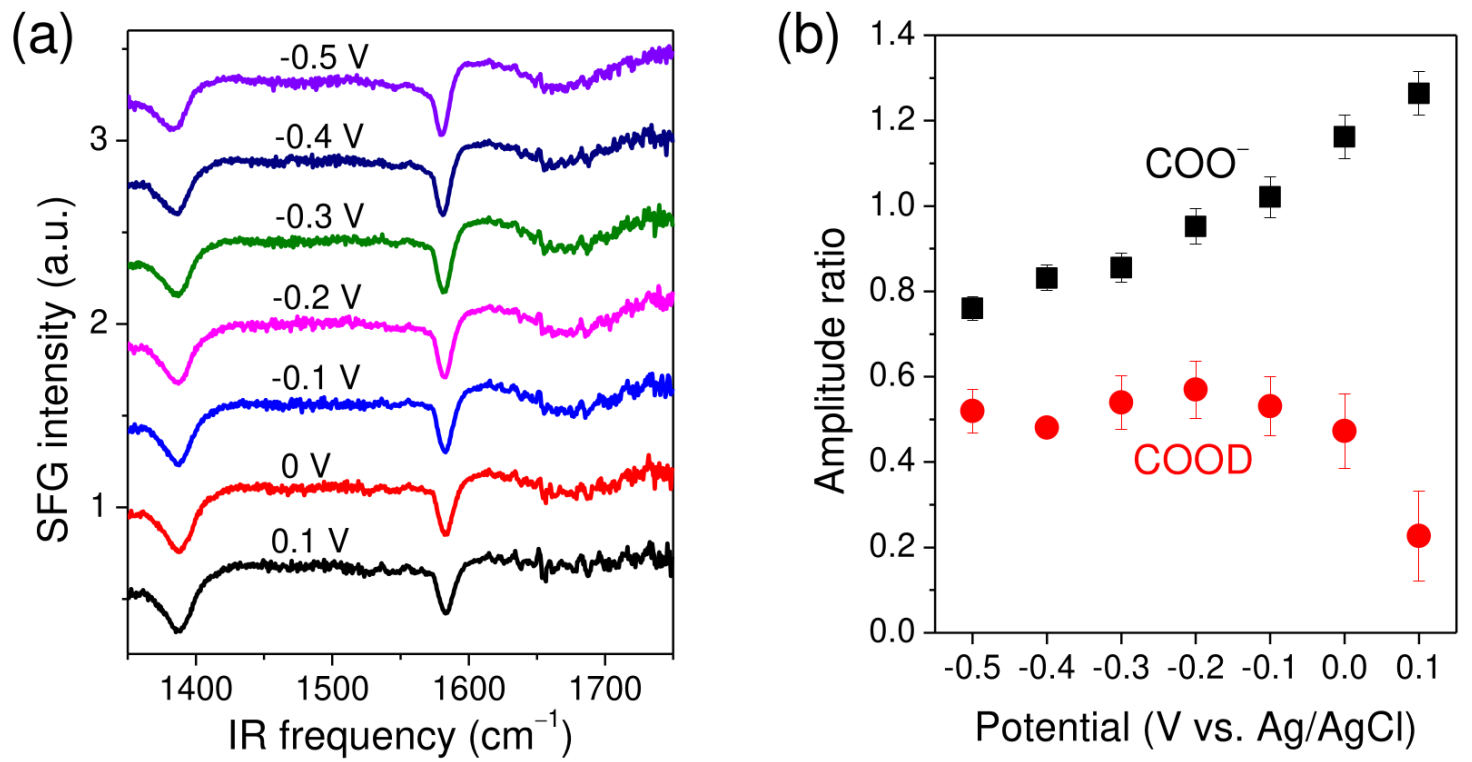

Figure S-5: Potential-dependent SFG spectra for 4-MBA SAM on Au at $\mathrm{pH} 6$. (a) SFG spectra at the indicated applied potential (vs. $\mathrm{Ag} / \mathrm{AgCl} 1 \mathrm{M} \mathrm{KCl}$ ). (b) Amplitude ratios $A_{\mathrm{COO}}^{\mathrm{ss}} / A_{\text {ring }}$ and $A_{\mathrm{COOD}} / A_{\text {ring }}$ as a function of applied potential.

of 4-MBA on a $\mathrm{Au}(111)$ surface as a function of the molecular tilt angle and coverage, we found that the molecule binds in a bridge position slightly shifted towards the hollow site. The most favorable binding energy was found for a $\mathrm{p}(2 \times 2) \mathrm{SAM}$ at a coverage of 0.25 with a molecular tilt angle of $48^{\circ}$ (Figure S-6), as has also been reported in previous studies [34-36]. Interestingly, the frequency of the $\mathrm{COOH}$ and $\mathrm{COO}^{-}$ groups depends sensitively on the title angle of the SAM layer. It is likely that observed pH-dependent $\mathrm{COO}^{-}$ frequency is indicative of a $\mathrm{pH}$-dependent binding geometry of the 4-MBA molecules in the deprotonated form, driven in part by the interaction of the charged $\mathrm{COO}^{-}$groups. More thoroughly described results and a comparison with other SAM geometries are provided in Section S2.4.

\section{S2.4. Adsorbate binding geometries}

To determine feasible SAM geometries, we scanned surface coverage configurations and tilt angles. The binding energy was calculated as

$$
\Delta E_{\text {bind }, x y}=\frac{E_{4-\mathrm{MBA} * x y}-E_{\mathrm{Au}, x y}-N \cdot\left(E_{4-\mathrm{MBA}}-\frac{1}{2} E_{\mathrm{H}_{2}}\right)}{N}
$$

where the $*$ denotes the adsorbed structure, and the subscript $x y$ represents the respective size of the unit cell plane parallel to the electrode surface in terms of $\mathrm{Au}$ atoms.

As shown in Figure S-6, a $\mathrm{p}(2 \times 2)$ SAM structure has been determined as the most stable. At lower coverages the binding energy becomes more positive, indicating that the interaction between the adsorbed 4-MBA species is attractive. This finding can in principle be attributed to both van der Waals interactions and $\mathrm{COH}-\mathrm{CO}$ hydrogen bonding. Due to the benzene ring in the backbone of the molecule, the proton of the $\mathrm{COOH}$ group is, however, forced to point away from the surface, reducing the stabilization of the SAM via hydrogen bonds substantially.

We are aware that the finding of a $\mathrm{p}(2 \times 2)$ geometry being the most stable is in a slight mismatch with results where the geometry of $\mathrm{c}(\sqrt{ } 3 \times 4)$ with two molecules per unit cell was reported in reference [34]. We attribute this finding to the differing exchange-correlation functional and the choice of definition of 

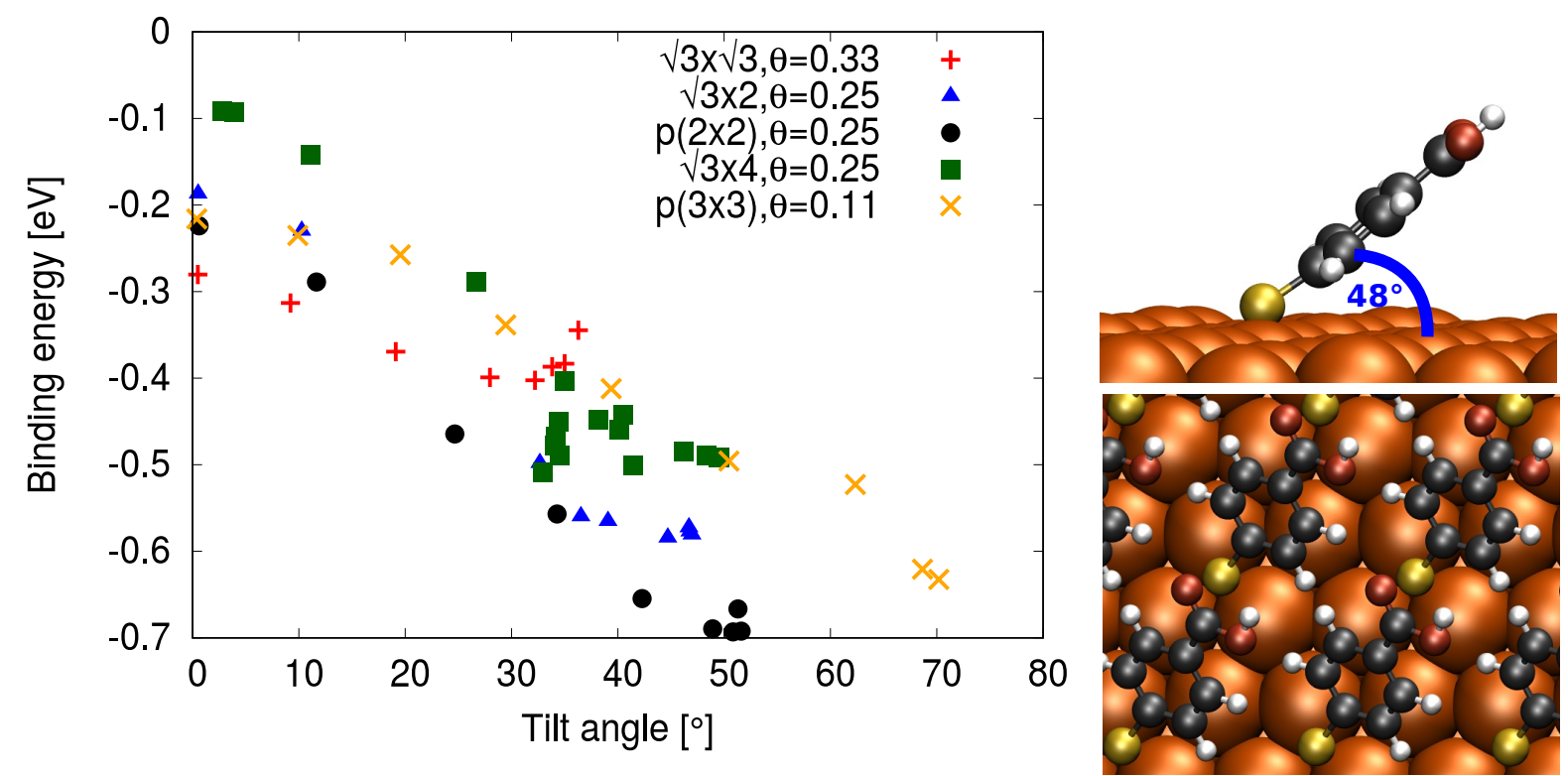

Figure S-6: Left panel: Binding energies of the 4-MBA SAM calculated by applying Equation (S.6). Right panels: Side and top view of the most stable SAM structure, where the tilt angle has been highlighted. In the side view only a single adsorbate is shown for clarity, while the actual SAM density can be seen in the top view.

the binding energy. However, since the experimental measurements were performed on polycrystalline Au substrates and, therefore, the theoretically studied surface geometries are certainly idealized, such slight changes in the SAM arrangement are beyond the scope of this study. In practice, we show the dependence of the $\mathrm{p} K_{\mathrm{a}}$ on potential for several candidate surface geometries in the text.

\section{S2.5. Simulated IR spectra}

We used DFT to calculate the surface IR spectra of both the protonated and anionic 4-MBA SAM, assuming the most stable coverage found in Section S2.4, in both a tilted and untilted configuration. To account for the selection rules customary to SFG measurements, the direction of the vibration is taken into account where only the transition dipole moment perpendicular to the electrode surface contribute to the IR intensities. Both structures were calculated with the same total number of electrons; that is, at a charge of 0 for the acid simulation and -1 for the anion simulation.

In the simulated spectrum of the protonated 4-MBA SAM, two distinct peaks have been identified corresponding to the ring vibration (8a) [37] at $1580 \mathrm{~cm}^{-1}$ and the $\mathrm{C}=\mathrm{O}$ stretch vibration $\left(\nu_{\mathrm{C}=\mathrm{O}}\right)$ at 1670 $\mathrm{cm}^{-1}$. While other infrared active vibrations exist in the frequency range of interest, their intensity is negligible, as a consequence of the orientation of their transition dipole moment. In the spectrum of the anionic SAM, $\nu_{\mathrm{C}=\mathrm{O}}$ is not present anymore. Instead, two new peaks appear at $1340 \mathrm{~cm}^{-1}$ and $1490 \mathrm{~cm}^{-1}$ corresponding to the symmetric and asymmetric $\mathrm{COO}^{-}$stretch vibration. The appearance of a peak related to the symmetric $\nu_{\mathrm{COO}^{-}}^{\mathrm{ss}}$ is in agreement with the experimental findings and consistent with the surface selection rules. However, the computed frequency of this mode is significantly lower than the observed band at $1380-1400 \mathrm{~cm}^{-1}$. This discrepancy may be caused by the $\mathrm{COO}^{-}$coverage-dependent peak shift shown in Figure S-3. The computed value $\left(1340 \mathrm{~cm}^{-1}\right)$ agrees better with the measured value $\left(\sim 1380 \mathrm{~cm}^{-1}\right)$ at low $\mathrm{COO}^{-}$surface fraction (at low $\mathrm{pH}$ ), but with increasing $\mathrm{COO}^{-}$fraction at higher $\mathrm{pH}$, the peak shift to higher energy, presumably caused by the interaction between $\mathrm{COO}^{-}$groups. It is quite possible that this 

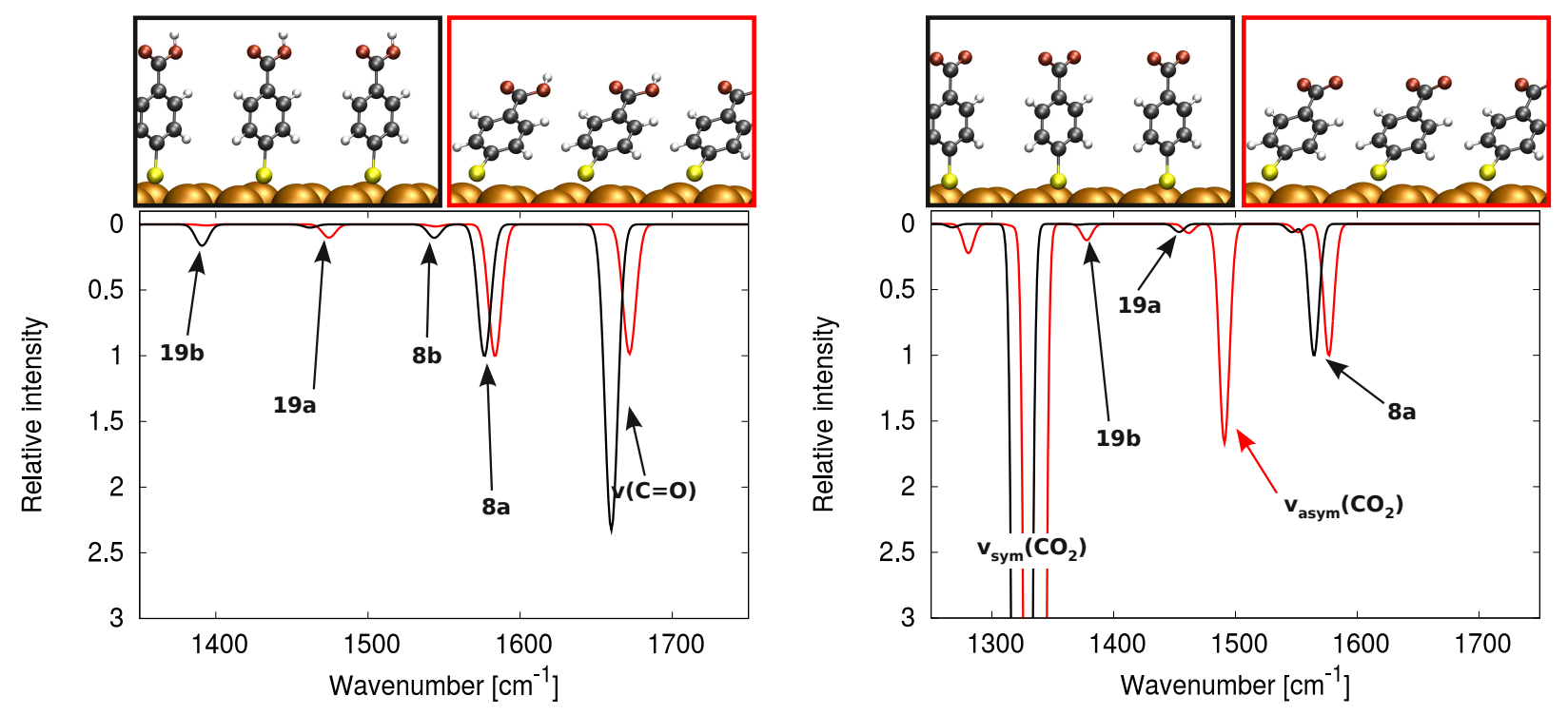

Figure S-7: Calculated IR spectra of the 4-MBA SAM in its protonated (left) and anionic (right) forms. Both the tilted SAM structure, shown in red, and the not tilted, shown in black, have been studied for both protonation states. The respective ring-vibration symmetries are given in terms of the Wilson notation. [37] All amplitudes have been normalized to the height of the $8 \mathrm{a}$ ring vibration, which has also been used as a reference in the experiments. An implicit solvent has been applied in all calculations.

interaction energy is not well captured in our calculation. The asymmetric $\nu_{\mathrm{COO}^{-}}^{\mathrm{as}}$ is weaker than the $\nu_{\mathrm{COO}}^{\mathrm{ss}}$ mode as can be seen in Figure S-4. Judging from the transition dipole perpendicular to the surface, this vibration should not lead to a significant intensity if the $\mathrm{COO}^{-}$group is in the plane with the phenyl ring of the molecule. However, we find that it is slightly rotated out of plane, which leads to the appearance of the respective peak in the IR spectrum. This is consistent with the SFG data (Figure S-4). However, it has to be emphasized again, that since the substrate consists of polycrystalline $\mathrm{Au}$, the actual geometry of the SAM might vary from the theoretically studied one. That is why we also studied the IR spectra calculated for the SAM in which the molecule is not tilted. In this spectrum, the asymmetric $\mathrm{COO}^{-}$vibration does not lead to a significant intensity anymore. This result is strong evidence for the experimentally studied SAMs to consist of 4-MBA molecules, whose tilt angle is lower than one would expect on a single crystal $\mathrm{Au}(111)$ surface. The lower tilt angle can be explained, for example, by surface reorganization including the creation of gold adatoms. For such structures, it has been shown that the tilt angle is reduced to $\sim 30^{\circ}[35]$.

\section{S2.6. Calculated potential profiles}

Figure S-8 shows the electrostatic potential averaged parallel to the electrode surface of the acidic and anionic SAMs. To facilitate the comparison, these profiles were generated with the same total number of electrons (i.e., an applied charge of 0 and -1 e for the acid and anion, respectively.) The structure plotted is the $\mathrm{p}(2 \times 2)$ without tilt. The protonated SAM exhibits a comparatively small surface dipole, which consists of two annihilating contributions, namely the $\mathrm{Au}-\mathrm{S}$ interface and the inherent dipole in the $\mathrm{COOH}$ group. After deprotonation, this dipole changes drastically. While the $\mathrm{Au}-\mathrm{S}$ interface dipole is still present, the anionic group represents a monopole. 


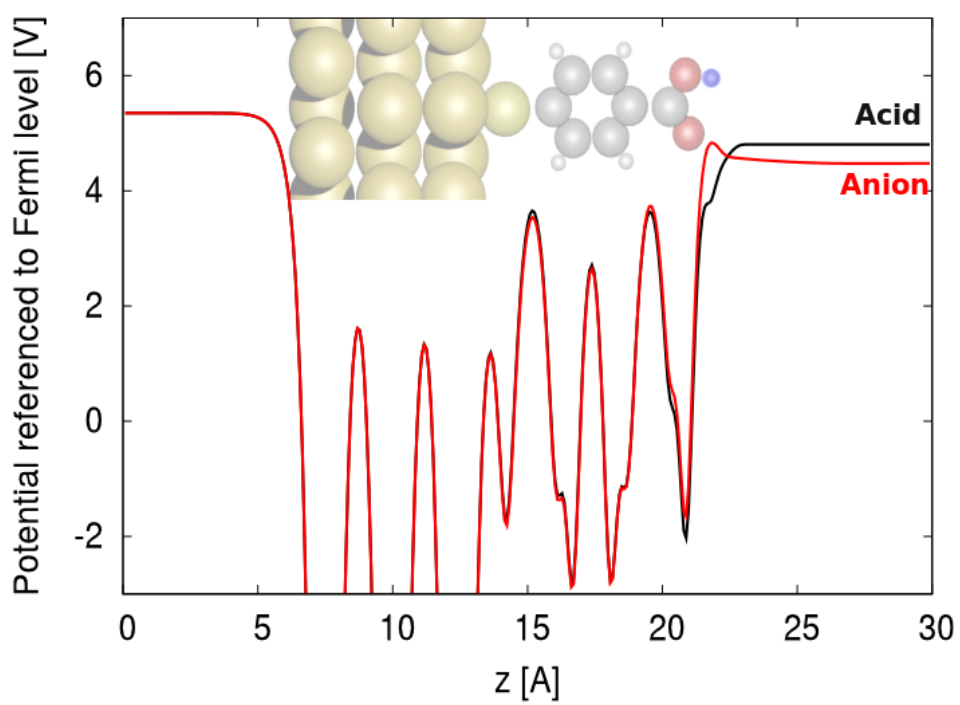

Figure S-8: Electrostatic potential of both the acidic and the anionic SAM structures plotted with an equivalent total number of electrons ( $q=0$ for acid, $q=-1$ for anion). The hydrogen dissociating is highlighted in blue.

\section{S2.7. Calculated $\mathrm{p} K_{\mathrm{a}}$ values}

Figure S-9(a) shows the calculated $\mathrm{p} K_{\mathrm{a}}^{\mathrm{ap}}$ resulting from applying the theory described in Section S1.5 to the two protonation states of the SAM. The $\mathrm{p} K_{\mathrm{a}}^{\mathrm{ap}}$ at a given potential is strongly dependent on the SAM geometry and coverage; however, all four slopes exhibit similar values and are in reasonable agreement with the experimentally measured value. (Note that the slope is indicative of the non-integer number of electrons transferred, as described in Section S1.5.) The untilted SAM's exhibit a lower $\mathrm{p} K_{\mathrm{a}}^{\mathrm{ap}}$, this is a consequence of the counter charge being able to both surround the anionic group and also penetrate the monolayer. As a consequence, the repulsive interaction between the anionic groups is quenched stabilizing the deprotonated form. Reducing the coverage with respect to the equilibrium coverage of 0.25 even increases this effect, where the $\mathrm{p} K_{\mathrm{a}}^{\mathrm{ap}}$ at $0 \mathrm{~V}$ has been determined as 3.8 and 3.3 for the shown coverages of 0.25 and 0.11 , respectively. For the tilted SAMs $\mathrm{p} K_{\mathrm{a}}^{\mathrm{ap}}$ is generally higher. This is a consequence of the more compact monolayer, where the solvent - and with it the counter charge - is not able to penetrate inside. As a consequence, the anionic structure is much less stabilized by the created double-layer, increasing $\mathrm{p} K_{\mathrm{a}}^{\text {ap }}$ of the SAM.

All shown curves in Figure S-9(a) exhibit a negative slope with the applied potential, indicating the anionic structure is more favored at more positive potentials. The value of the slope varies depending on the SAM geometry and coverage. While the tilted structures resulted in a potential response of -3.3 and $-3.6 \mathrm{~V}^{-1}$ (with $n \approx-0.20$ and -0.26 ) for a coverage of 0.25 and 0.11 , respectively, the SAM without tilt leads to substantially lower slopes of -2.4 and $-1.5 \mathrm{~V}^{-1}$ for the same coverages, with corresponding lower $n$ of -0.14 and -0.09 , respectively. ( $n$ is the non-integer number of electrons involved in the reaction, as defined in reaction (S.3).) The source of this difference can again be found in the penetration of the counter-charge into the SAM. By penetrating the SAM structure, the overall net charge in the interface region is reduced. As a consequence, the double layer which is created on top of the slab will be weakened.

Same as in the case of the simulated IR-spectra (Section S2.5), the good agreement between the simulated SAM structures without tilt and the experimental results indicates that the tilted SAM structure determined as the most stable on $\mathrm{Au}(111)$, does not correspond to the actual SAMs present in the experimental studies. As a consequence of this rather low potential response of the untilted monolayers, we expect the effect of an applied potential to be only significant at $\mathrm{pH}$ values close to the $\mathrm{p} K_{\mathrm{a}}^{\mathrm{ap}}$ of the SAM. As shown in Figure 

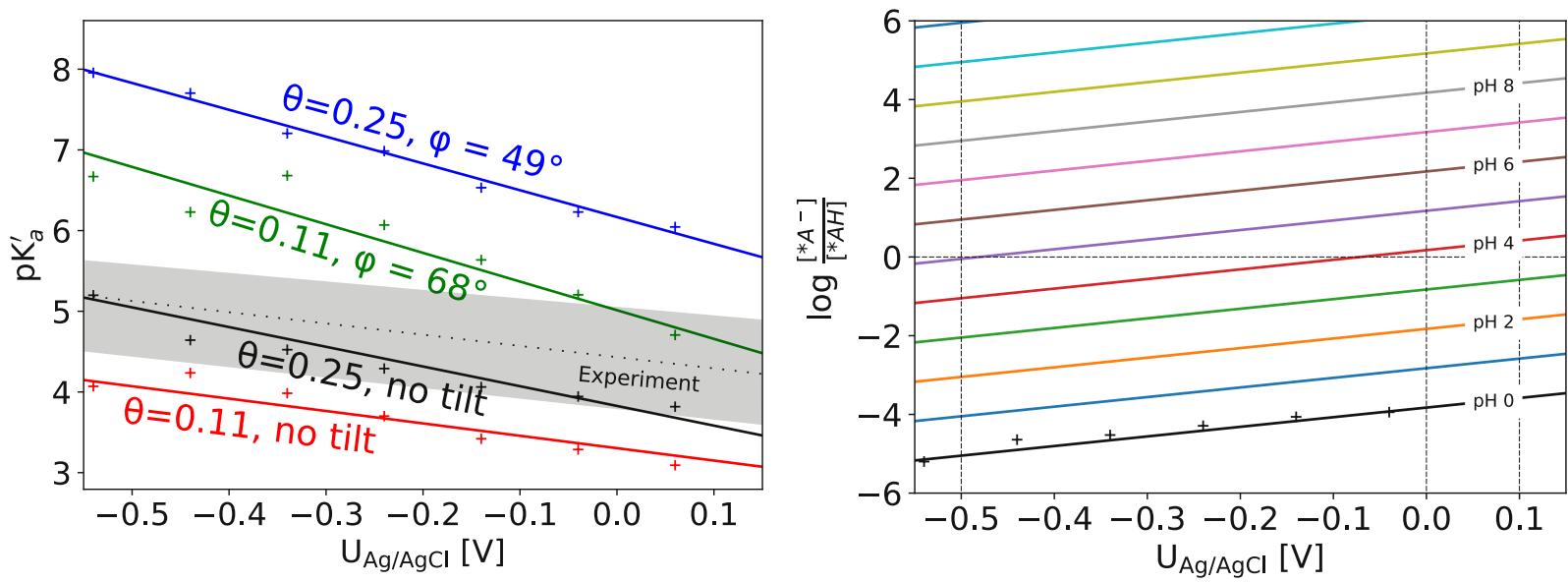

Figure S-9: (a) Calculated $\mathrm{p} K_{\mathrm{a}}^{\mathrm{ap}}$ and (b) degree of dissociation versus the applied potential. The value for the $\mathrm{pH}$ where the lines cross the horizontal line drawn at $\log \left[* \mathrm{~A}^{-}\right] /[* \mathrm{AH}]=0$ represent $\mathrm{p} K_{\mathrm{a}}^{\mathrm{ap}}$ at the given potential. The vertical dashed lines indicate $-0.5 \mathrm{~V}, 0 \mathrm{~V}, 0.1 \mathrm{~V}$ on a $\mathrm{Ag} / \mathrm{AgCl}$ scale (taken as $0 \mathrm{~V}_{\mathrm{SHE}}+0.2$ $\left.\mathrm{V}=4.64 \mathrm{~V}_{\mathrm{abs}}\right)$.

S-9(b), the determined $\mathrm{pH}$ of half-dissociation only varies by 1.5 in the scanned potential range, consistent with experimental results.

The shape and strength of the electrochemical double layer in the SJ method are, of course, drastically approximated, by exchanging actual counterions by a continuum charge. Therefore, quantitative interpretations should be made with caution. However, a qualitative interpretation allows an understanding of the sign in the slope of $\mathrm{p} K_{\mathrm{a}}^{\mathrm{ap}}$ on an atomistic level, which is consistent with the macroscopic picture given in earlier models $[38,39]$. We hope that we can inspire future studies applying more advanced models for the electrochemical interface, in order to fully elucidate the effect of an electrode potential on the degree of protonation for acidic SAMs.

\section{S2.8. Comparison between 2-MBA and 4-MBA}

The model based on Gauss's Law, which is summarized in the main text and described in detail in Section S3, provides an intuitive understanding for the non-integer electron transfer that results from the acid-dissociation reaction. It also makes qualitative predictions: for example, it suggests that if the point of the charge were moved closer to the electrode, then the amount of non-integer electron transfer $(n)$ required to keep the potential constant would be greater; this also implies that the slope of $\mathrm{p} K_{\mathrm{a}}$ versus potential would be greater. Interestingly, the mercaptobenzoic acid molecule lends itself to such a question: if the functional group were moved from the para position (4-MBA) to the ortho position (2-MBA), then the charge would be located closer to the electrode surface and we would have a natural test of the model prediction. Ma \& Harris [32] have performed such experiments on the 2-MBA system, albeit with a silver, rather than gold, substrate, and indeed measured a higher slope of the $\mathrm{p} K_{\mathrm{a}}-$ potential relation, on the order of $-10 \mathrm{~V}^{-1}$.

We also examined this system with constant-potential electronic structure calculations. To facilitate comparisons with both our other calculations and the experimental measurements, we performed these calculations on both $\mathrm{Au}$ and $\mathrm{Ag}$ surfaces. To keep this simple while still being able to estimate the slope, we only examined the no-tilt, low coverage situation for 2-MBA; also, we assumed that the vibrational modes on the $\mathrm{Au}$ and $\mathrm{Ag}$ surfaces were the same, and only performed the normal-mode analysis on Au. (Note that in the investigation of 4-MBA, we found the intercepts to vary strongly with binding geometry, but the 

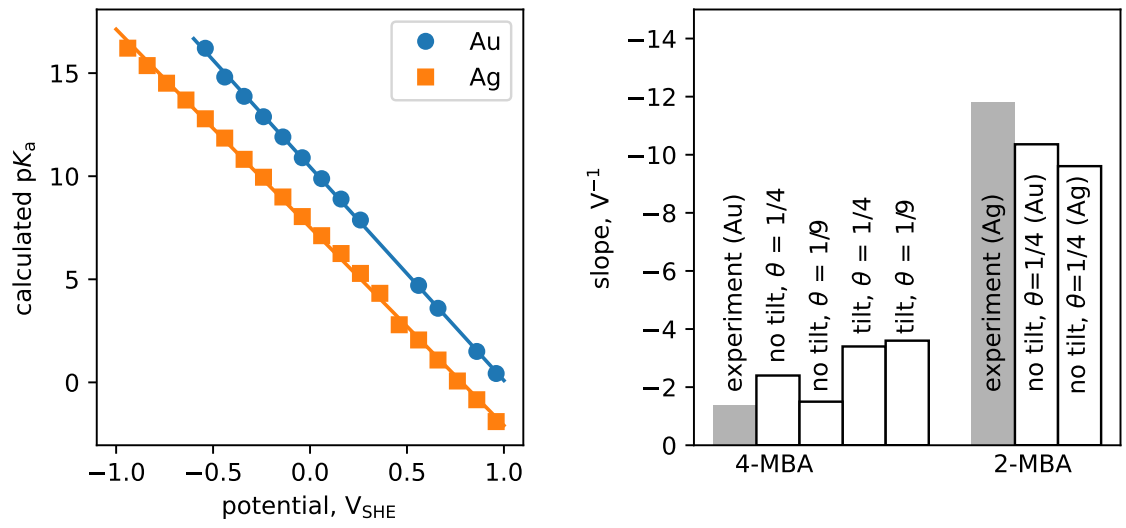

Figure S-10: Comparison to 2-MBA. Left: Calculated $\mathrm{p} K_{\mathrm{a}}$ values for 2-MBA on both $\mathrm{Au}$ and $\mathrm{Ag}$ surfaces. Right: Comparison between experimentally measured $\mathrm{p} K_{\mathrm{a}}-$ potential slopes and those calculated under different assumptions. Experimental data on 2-MBA from Ma \& Harris [32].

slopes to all be similar.) The results of these calculations are shown in Figure S-10. The slopes on both the $\mathrm{Au}$ and $\mathrm{Ag}$ substrates were much higher than on 4-MBA, as would be predicted by a Gauss's Law model, and are again in close agreement with the experiments.

We can also infer the non-integer electron transfer $n$, from equation (S.3), from our calculations by comparing the number of electrons in the calculations of the ion and acid forms of 2-MBA at a specified potential. We found $n$ to be about -0.6 for 2-MBA, as compared to about -0.1 for 4-MBA, in qualitative agreement with the Gauss-law prediction.

\section{S3. Gauss's law model of charge--potential relation}

Here, we use a formalism based on Gauss's law to understand the relationship between charge and potential, and specifically how we can expect the $\mathrm{p} K_{\mathrm{a}}$ to vary with the potential of the electrode. We derive this in two different frameworks, and show the equivalence of the two interpretations.

\section{S3.1. $\mathrm{p} K_{\mathrm{a}}$ in the open-electron interpretation}

The $\mathrm{p} K_{\mathrm{a}}$ describes a reaction equilibrium, which is dictated by $\Delta G_{\mathrm{rxn}}$, the free-energy change of reaction. We write the reaction in an electronically grand canonical fashion as:

$$
* \mathrm{AH}+n \mathrm{e}^{-} \stackrel{\phi}{\longrightarrow} * \mathrm{~A}^{(1+n)-}+\mathrm{H}^{+}
$$

where the potential $\phi$ is held constant by the circuitry of the electrode. Note that in the absence of an electrode, $n$ would equal 0 , and we are left with our standard acid-dissociation reaction. The equilibrium constant of the above reaction is defined to be:

$$
K_{\mathrm{a}}=\frac{\left[* \mathrm{~A}^{(1+n)-}\right]\left[\mathrm{H}^{+}\right]}{[* \mathrm{AH}]\left[\mathrm{e}^{-}\right]^{n}}=\exp \left\{\frac{-\Delta G_{\mathrm{rxn}}^{\circ}}{k_{\mathrm{B}} T}\right\}
$$

where the terms in brackets are properly activities, not concentrations, each defined to be 1 at the reference state used in $\Delta G_{\mathrm{rxn}}^{\circ}$. The $\mathrm{p} K_{\mathrm{a}}$ is then

$$
\mathrm{p} K_{\mathrm{a}}=-\log _{10} \exp \left\{\frac{-\Delta G_{\mathrm{rxn}}^{\circ}}{k_{\mathrm{B}} T}\right\}=\frac{\Delta G_{\mathrm{rxn}}^{\circ}}{\ln 10 \cdot k_{\mathrm{B}} T}
$$


The free energy change of the reaction, $\Delta G_{\mathrm{rxn}}^{\circ}$, is a function of potential $\phi$ and is written as:

$$
\Delta G_{\text {rxn }}^{\circ}(\phi)=\mu^{\phi}\left[* \mathrm{~A}^{(1+n)-}\right]+\mu^{\circ}\left[\mathrm{H}^{+}\right]-\mu^{\phi}[* \mathrm{AH}]-n \mu^{\phi}\left[\mathrm{e}^{-}\right](\phi)
$$

where $\mu^{\phi}$ is the chemical potential of the indicated species at potential $\phi$. When performing electronic structure calculations, the first and third species would be calculated at specified potential $(\phi)$ with a method such as the solvated jellium approach. [13] The thermodynamic reservoir for protons is determined by the bulk $\mathrm{pH}$ and is unaffected by the potential. The last term is the electron that is at the potential supplied by the circuitry, and it can be re-written as

$$
\mu^{\phi}\left[\mathrm{e}^{-}\right]=-e \phi
$$

Here, we are defining the reference energy to be $\mu\left[\mathrm{e}^{-}\right] \equiv 0$ at $\phi=0$. $e$ is the (positive) charge on an electron if we are working in units of $\mathrm{eV}$ and $\mathrm{V}$.

From the experimental results, we are most interested in the slope (that is, the potential dependence of the $\mathrm{p} K_{\mathrm{a}}$ ) which is then:

$$
\frac{d \mathrm{p} K_{\mathrm{a}}}{d \phi} \approx \frac{n e}{\ln 10 \cdot k_{\mathrm{B}} T}
$$

At room temperature, this works out to be $16.9 n$, where the coefficient has units of $\mathrm{V}^{-1}$. With an experimental slope of about $-1.4 \mathrm{~V}^{-1}$ as seen in this work, this gives $n$ to be about -0.1 electrons.

\section{S3.2. $\quad \mathrm{p} K_{\mathrm{a}}$ in the point-charge interpretation}

In the point-charge interpretation, we write the chemical equilibrium ignoring any extra electrons that must be supplied by the circuit; that is, the reaction is simply

$$
* \mathrm{AH} \longrightarrow * \mathrm{~A}^{-}+\mathrm{H}^{+}
$$

Now the $\Delta G_{\mathrm{rxn}}^{\circ}$ equation is written as

$$
\Delta G_{\mathrm{rxn}}^{\circ}(\phi)=\mu\left[* \mathrm{~A}^{-}\right](\phi)+\mu\left[\mathrm{H}^{+}\right]-\mu[* \mathrm{AH}]
$$

There are two charged species. As before, the thermodynamic reservoir of the proton $\left(\mathrm{H}^{+}\right)$is determined by the bulk $\mathrm{pH}$ and is unaffected by the electrode potential. The deprotonated acid $* \mathrm{~A}^{-}$is close enough to the surface to feel at least part of the potential change. Let's assume the deprotonated species' chemical potential can be written as a function of potential as:

$$
\mu\left[* \mathrm{~A}^{-}\right](\phi)=\mu\left[* \mathrm{~A}^{-}\right]\left(\phi^{0}\right)-e \delta \phi
$$

where $\delta$ is the fraction of the potential change $\left(\phi-\phi^{0}\right)$ felt at the "point" of the charge of $* \mathrm{~A}^{-}$. This means the slope will be:

$$
\frac{d \mathrm{p} K_{\mathrm{a}}}{d \phi}=\frac{-\delta e}{\ln 10 \cdot k_{\mathrm{B}} T}
$$

which is a nearly identical form as earlier, but with a very different interpretation. In this approach, we'd deduce from the experimental slope that $\delta \approx 0.1$; that is, at the point of the negative charge in $* \mathrm{~A}^{-}$about $10 \%$ of the potential change is felt.

\section{S3.3. Gauss's law equivalence}

Equations (S.7) and (S.8) are functionally equivalent, but are derived and interpreted in contrasting frames of reference. Here, we will use a Gauss's law treatment to show the equivalence of these two formalisms, at least within a simple model system. 

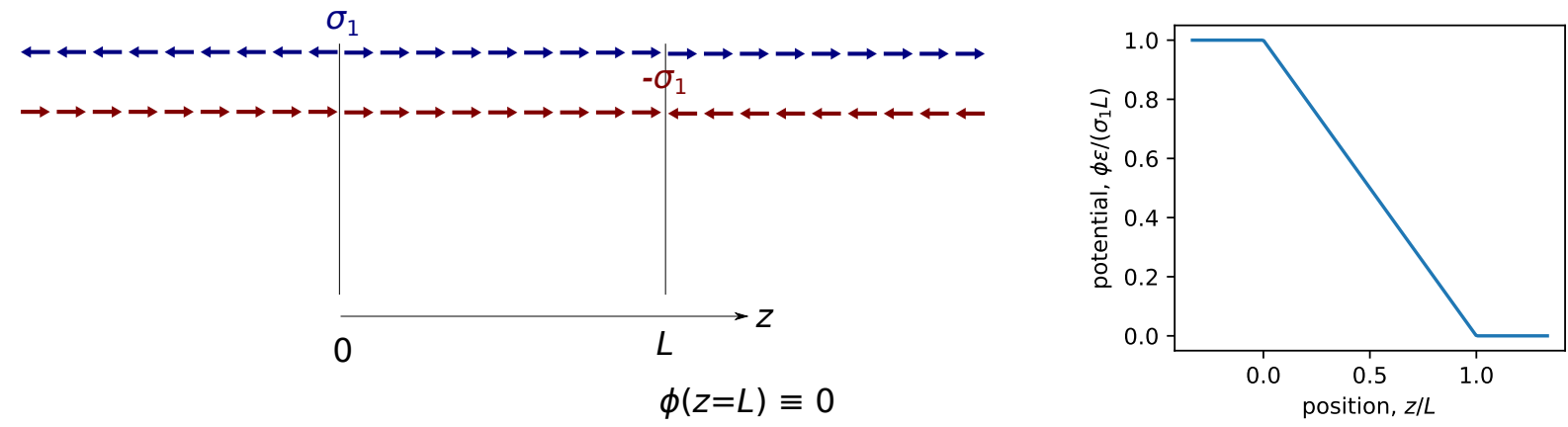

Figure S-11: (Left) Simple one-dimensional model of a charged surface. (Right) The potential that results from integrating the net field; variables changed to dimensionless form.

Gauss's Law. Gauss' law states that the total of the electric flux out of a closed surface is equal to the charge enclosed $(Q)$ divided by the permittivity $(\epsilon)$. For an infinite plane with uniform charge density $\sigma=Q / A$, the electric field emanating normal to this plane is:

$$
E=\frac{\sigma}{2 \epsilon}
$$

This field emanates infinitely, in both directions from the plane. Fields from multiple sources are additive. Recall that the field is the (negative) gradient of the electric potential $\phi$, so in the one-dimensional case (normal to the plane) $E=-d \phi / d z$.

First model: surface charging. Let's start with a simple capacitor, as a model of our electrified surface. (We can take this to be either a bare surface or a surface with the protonated molecule present, HA*.) This is sketched in Figure S-11. The physical analogy to our system is: the plane at $z=0$ corresponds to the metal surface, while $z=L$ corresponds to the countercharge (that is, the jellium). In this simple model we take them each to be planes of infinite area (in the $x y$ direction) and infinitesimal thickness(in the $z$ direction). $\sigma_{1}$ is the uniform charge density; that is, the charge per unit area. It is equal and opposite in this case.

The field emanating from the planar charge $\sigma_{1}$, located at $z=0$ is $\sigma_{1} /(2 \epsilon)$; here, we have taken the convention that the field arrows in the figure point away from the charge. Similarly, the field emanating from the planar charge $-\sigma_{1}$, located at $z=L$, is $-\sigma_{1} /(2 \epsilon)$. Because of the negative sign, the field arrows point in towards the charged plane at $z=L$.

We can find the net field by adding these fields in all space. We can see that the field is then:

$$
E(z)=\left\{\begin{array}{cl}
0, & z<0 \\
\frac{\sigma_{1}}{\epsilon}, & 0<z<L \\
0, & z>L
\end{array}\right.
$$

This is, of course, a simple capacitor, and we see that the field cancels outside of the capacitor and has a constant field of $\sigma_{1} / \epsilon$ within. Here we will define the potential to be 0 at $z=L$, and the potential at $z=0$ is just found by integrating the field.

$$
\phi(z=0)=\frac{\sigma_{1} L}{\epsilon}
$$

In this model of an electrode, we can adjust the potential (at $z=0$ ) by varying $\sigma_{1}$; that is, the potentiostat injects or removes electrons to hold the potential constant. This potential as a function of position is also plotted in Figure S-11. 

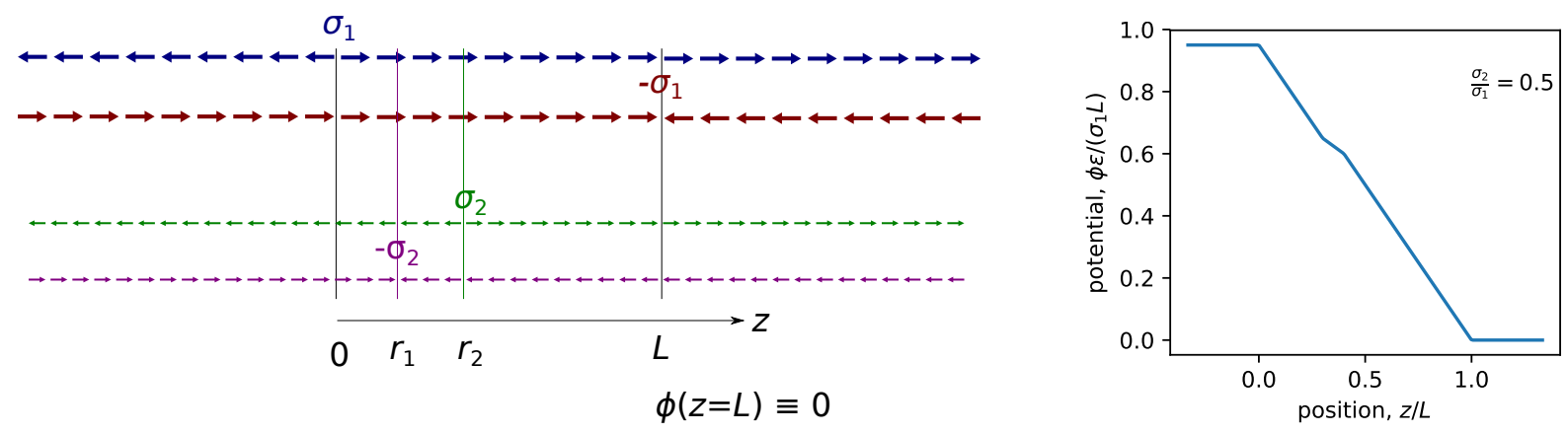

Figure S-12: (Left) A "surface dipole" added above the charged surface. (Right) The potential that results from integrating the net field.

Aside: neutral adsorbates and a "surface dipole". Before examining $\mathrm{p} K_{\mathrm{a}}$, we will first look at how a neutral adsorbate can change the number of electrons needed to keep an electrode at a specified potential. In Figure S-12 we show the addition of two planes of opposite charge that make up a dipole in the direction normal to the surface. This surface-normal dipole might be created by a monolayer of CO adsorbates (with slightly positive C's and slightly negative O's), water in the H-up or H-down configuration, or even an $\mathrm{H}$ adsorbate (where presumably the electron is localized more in the sigma bond, so there is vertical charge separation).

Note that this surface dipole looks like a second capacitor inserted into the middle of the first. Now the field profile will be:

$$
E(z)=\left\{\begin{array}{cl}
0, & z<0 \\
\frac{\sigma_{1}}{\epsilon}, & 0<z<r_{1} \\
\frac{\sigma_{1}}{\epsilon} \frac{\sigma_{2}}{\epsilon}, & r_{1}<z<r_{2} \\
\frac{\sigma_{1}}{\epsilon}, & r_{1}<z<L \\
0, & z>L
\end{array}\right.
$$

That is, the field looks like the previous example, except in the region between $r_{1}$ and $r_{2}$, where an extra term is added to it. When we integrate we get:

$$
\phi(z=0)=\frac{\sigma_{1}}{\epsilon} L-\frac{\sigma_{2}}{\epsilon}\left(r_{2}-r_{1}\right)
$$

So the potential at the surface $(z=0)$ is not the same as in the previous example, due to the surface dipole or capacitor. The potential profile is also shown in the figure. If the dipole is fixed, then the only means we have of tuning the potential back to its previous value is by adjusting $\sigma_{1}$. In an atomistic simulation, we can do this by changing the number of electrons in the simulation; in an experiment, the potentiostat also keeps the surface potential constant by inserting or removing electrons into the circuit in the form of current. This helps us to understand why we need to slightly adjust the charging in constant-potential simulations from one configuration to the next, as these small dipoles will be different.

Interestingly, we also see that the perturbation in equation (S.10) is entirely due to the dipole, meaning the amount of charge and the separation of that charge from itself. It has nothing to do with where the dipole is placed within the domain of the problem.

Adding a charged species. Now we deal with the case in the manuscript in which there is a charged species at some point above the electrode surface, at $z=r$; this will be balanced by an equal-and-opposite charge at $z=L$, as shown in Figure S-13. Since the charged species forms a monolayer, a planar charge density $\left(\sigma_{2}\right)$ is again a reasonable model. By an identical analysis to the previous example, we can deduce the field to be: 

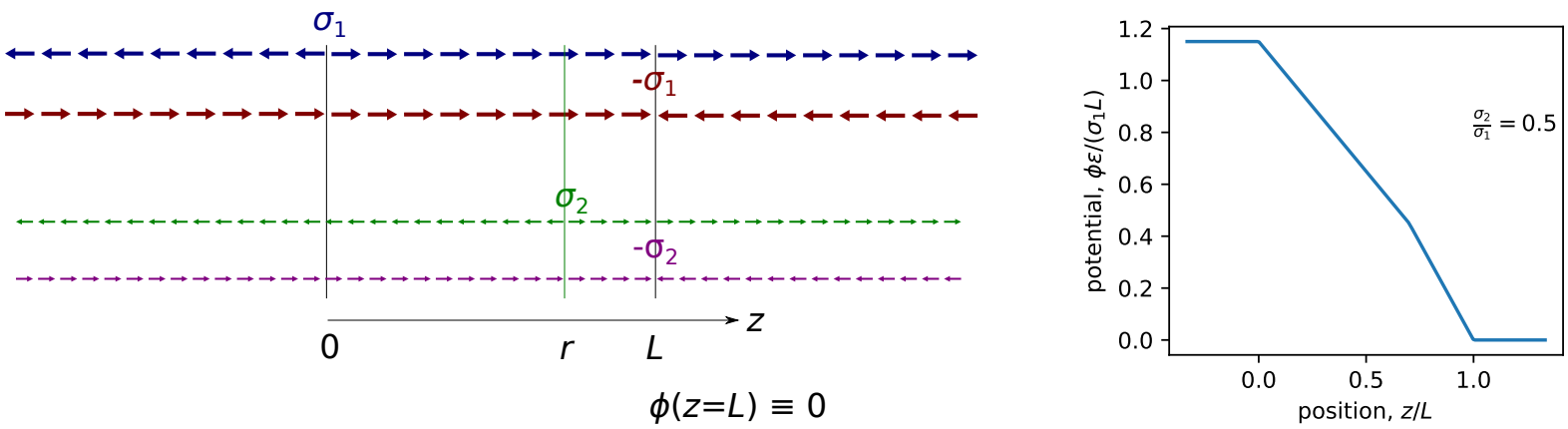

Figure S-13: (Left) A charged species bound to the surface. (Right) The potential that results from integrating the net field.

$$
E(z)=\left\{\begin{array}{cl}
0, & z<0 \\
\frac{\sigma_{1}}{\epsilon}, & 0<z<r \\
\frac{\sigma_{1}}{\epsilon}+\frac{\sigma_{2}}{\epsilon}, & r<z<L \\
0, & z>L
\end{array}\right.
$$

Integrating gives

$$
\phi(z=0)=\frac{\sigma_{1}}{\epsilon} L+\frac{\sigma_{2}}{\epsilon}(L-r)
$$

So here we can see that the presence of this charge - although it is balanced by an opposite charge (in the jellium) - induces a change in the surface potential at $z=0$. Practically, our potentiostat would need to adjust the surface charging $\sigma_{1}$ to make up for this by adding or removing electrons.

Interestingly, we can already see that the two interpretations (of Sections S3.1 and S3.2) are becoming consistent if we examine the limiting cases. If we move the charge to the right, such that $r \rightarrow L$, we are in a region where $100 \%$ of the potential has dropped off before it gets to the charged species; correspondingly, we would see that we would need to supply no "extra" electrons in order to hold the potential constant. (We would not need to adjust $\sigma_{1}$ in this model.) As we move in the other direction, the charge "feels" more of any changes that are made to the potential, and correspondingly the number of "extra" electrons needed to hold the potential constant will increase.

Linking the two explanations. To understand if the explanations of Sections S3.1 and S3.2 are equivalent in this model, then we must find how to express $n$ of equation (S.7) and $\delta$ of equation (S.8) in the terms above. To find $n$, we ask the question: how much do we need to adjust $\sigma_{1}$ in order to keep the potential constant after the charge $\sigma_{2}$ is introduced? We'll use $\sigma_{1}$ and $\sigma_{1}^{\prime}$ as follows:

- $\sigma_{1}$ : The charging on the original system where $\sigma_{2}$ was absent, that resulted in the potential $\phi(z=0)$.

- $\sigma_{1}^{\prime}$ : The new charge needed, after $\sigma_{2}$ is introduced, that keeps the $z=0$ potential at the same value as when $\sigma_{2}$ was absent.

To find the new charge needed, we just equate equations (S.9) and (S.11):

$$
\begin{gathered}
\frac{\sigma_{1}}{\epsilon} L=\frac{\sigma_{1}^{\prime}}{\epsilon} L+\frac{\sigma_{2}}{\epsilon}(L-r) \\
\sigma_{1}^{\prime}-\sigma_{1}=\sigma_{2} \frac{L-r}{L}
\end{gathered}
$$


Here again, we see that as the dipole moves farther in the field away from the surface, the amount of extra charge required $\left(\sigma_{1}^{\prime}-\sigma_{1}\right)$ goes to zero. Tying the charges back to Section S3.1, $\sigma_{1}^{\prime}-\sigma_{1}=n e$, and $\sigma_{2}=-e$, therefore:

$$
n=-\frac{L-r}{L}
$$

which gives the extra electrons necessary in this model to hold the potential constant when the charge in $* \mathrm{~A}^{-}$is introduced.

The other interpretation deals not in position but in potential. Specifically it asks: how much of an applied change in potential does our charge "feel"? Therefore, $\delta$ is defined as the partial derivative:

$$
\delta \equiv\left(\frac{\partial \phi(r)}{\partial \phi(0)}\right)_{r, L, \sigma_{2}}
$$

We can evaluate this partial derivative by first writing an expression for $\phi(r)$ :

$$
\begin{gathered}
\phi(r)=\phi(0)-\frac{\sigma_{1}^{\prime}}{\epsilon} r \\
\left(\frac{\partial \phi(r)}{\partial \phi(0)}\right)_{r, L, \sigma_{2}}=1-\frac{r}{\epsilon}\left(\frac{\partial \sigma_{1}^{\prime}}{\partial \phi(0)}\right)_{r, L, \sigma_{2}}
\end{gathered}
$$

The remaining partial derivative can come from writing $\phi(0)$ in terms of charges (integrating from $\phi(L) \equiv 0)$ :

$$
\begin{gathered}
\phi(0)=\frac{\sigma_{2}}{\epsilon}(L-r)+\frac{\sigma_{1}^{\prime}}{\epsilon} L \\
\left(\frac{\partial \phi(0)}{\partial q_{1}^{\prime}}\right)_{r, L, \sigma_{2}}=\frac{L}{\epsilon}
\end{gathered}
$$

Therefore we find:

$$
\left(\frac{\partial \phi(r)}{\partial \phi(0)}\right)_{r, L, \sigma_{2}}=1-\frac{r}{L}
$$

or

$$
\delta=\frac{L-r}{L}
$$

Thus, we han conclude that

$$
\delta=-n
$$

and by comparing to equations (S.7) and (S.8) we see that the two interpretations are identical, at least within this model.

\section{References}

[1] Krężel, A.; Bal, W. A formula for correlating $\mathrm{p} K_{\mathrm{a}}$ values determined in $\mathrm{D}_{2} \mathrm{O}$ and $\mathrm{H}_{2} \mathrm{O}$. J. Inorg. Biochem. 2004; 98, $161-166$.

[2] Ge, A.; Videla, P.E.; Lee, G.L.; Rudshteyn, B.; Song, J.; Kubiak, C.P.; Batista, V.S.; Lian, T. Interfacial Structure and Electric Field Probed by in Situ Electrochemical Vibrational Stark Effect Spectroscopy and Computational Modeling. J. Phys. Chem. C 2017; 121, 18674-18682. 
[3] Clark, M.L.; Ge, A.; Videla, P.E.; Rudshteyn, B.; Miller, C.J.; Song, J.; Batista, V.S.; Lian, T.; Kubiak, C.P. $\mathrm{CO}_{2}$ Reduction Catalysts on Gold Electrode Surfaces Influenced by Large Electric Fields. J. Am. Chem. Soc. 2018; 140, 17643-17655.

[4] Eisenthal, K.B. Liquid Interfaces Probed by Second-Harmonic and Sum-Frequency Spectroscopy. Chem. Rev. 1996; 96, 1343-1360.

[5] Shen, Y.R. Fundamentals of Sum-Frequency Spectroscopy. Cambridge University Press, Cambridge 2016.

[6] Strazdaite, S.; Meister, K.; Bakker, H.J. Reduced Acid Dissociation of Amino-Acids at the Surface of Water. J. Am. Chem. Soc. 2017; 139, 3716-3720.

[7] Yamaguchi, S.; Bhattacharyya, K.; Tahara, T. Acid-base equilibrium at an aqueous interface: PH spectrometry by heterodyne-detected electronic sum frequency generation. J. Phys. Chem. C 2011; $115,4168-4173$.

[8] Yamaguchi, S.; Kundu, A.; Sen, P.; Tahara, T. Communication: Quantitative estimate of the water surface $\mathrm{pH}$ using heterodyne-detected electronic sum frequency generation. J. Chem. Phys. 2012; 137, 151101.

[9] Talley, C.E.; Jusinski, L.; Hollars, C.W.; Lane, S.M.; Huser, T. Intracellular pH Sensors Based on Surface-Enhanced Raman Scattering. Anal. Chem. 2004; 76, 7064-7068.

[10] Wang, F.; Widejko, R.G.; Yang, Z.; Nguyen, K.T.; Chen, H.; Fernando, L.P.; Christensen, K.A.; Anker, J.N. Surface-Enhanced Raman Scattering Detection of $\mathrm{pH}$ with Silica-Encapsulated 4-Mercaptobenzoic Acid-Functionalized Silver Nanoparticles. Anal. Chem. 2012; 84, 8013-8019.

[11] Pienpinijtham, P.; Vantasin, S.; Kitahama, Y.; Ekgasit, S.; Ozaki, Y. Nanoscale pH profile at a solution/solid interface by chemically modified tip-enhanced raman scattering. J. Phys. Chem. C 2016; $120,14663-14668$.

[12] Zhuang, X.; Miranda, P.B.; Kim, D.; Shen, Y.R. Mapping molecular orientation and conformation at interfaces by surface nonlinear optics. Phys. Rev. B 1999; 59, 12632-12640.

[13] Kastlunger, G.; Lindgren, P.; Peterson, A.A. The controlled-potential simulation of elementary electrochemical reactions: proton discharge on metal surfaces. J. Phys. Chem. C 2018; 122, 12771-12781.

[14] Mortensen, J.J.; Hansen, L.B.; Jacobsen, K.W. Real-space grid implementation of the projector augmented wave method. Phys. Rev. B 2005; 71, 035109.

[15] Enkovaara, J.; Rostgaard, C.; Mortensen, J.J.; Chen, J.; Dułak, M.; Ferrighi, L.; Gavnholt, J.; Glinsvad, C.; Haikola, V.; Hansen, H.A.; Kristoffersen, H.H.; Kuisma, M.; Larsen, A.H.; Lehtovaara, L.; Ljungberg, M.; Lopez-Acevedo, O.; Moses, P.G.; Ojanen, J.; Olsen, T.; Petzold, V.; Romero, N.A.; StausholmMøller, J.; Strange, M.; Tritsaris, G.A.; Vanin, M.; Walter, M.; Hammer, B.; Häkkinen, H.; Madsen, G.K.H.; Nieminen, R.M.; Nørskov, J.K.; Puska, M.; Rantala, T.T.; Schiøtz, J.; Thygesen, K.S.; Jacobsen, K.W. Electronic structure calculations with GPAW: a real-space implementation of the projector augmented-wave method. J. Phys.: Condens. Matter 2010; 22, 253202.

[16] Held, A.; Walter, M. Simplified continuum solvent model with a smooth cavity based on volumetric data. J. Chem. Phys. 2014; 141, 174108.

[17] Lindgren, P.; Kastlunger, G.; Peterson, A.A. A Challenge to the G $\sim 0$ Interpretation of Hydrogen Evolution. ACS Catal. 2019; 10, 121-128.

[18] Lindgren, P.; Kastlunger, G.; Peterson, A.A. Scaled and Dynamic Optimizations of Nudged Elastic Bands. J. Chem. Theory Comput. 2019; 15, 5787-5793. PMID: 31600078. 
[19] Wellendorff, J.; Lundgaard, K.T.; Møgelhøj, A.; Petzold, V.; Landis, D.D.; Nørskov, J.K.; Bligaard, T.; Jacobsen, K.W. Density functionals for surface science: Exchange-correlation model development with Bayesian error estimation. Phys. Rev. B 2012; 85, 235149.

[20] Larsen, A.H.; Kuisma, M.; Löfgren, J.; Pouillon, Y.; Erhart, P.; Hyldgaard, P. libvdwxc: a library for exchange-correlation functionals in the vdW-DF family. Modell. Simul. Mater. Sci. Eng. 2017; 25, 065004 .

[21] Rossmeisl, J.; Chan, K.; Ahmed, R.; Tripković, V.; Björketun, M.E. pH in atomic scale simulations of electrochemical interfaces. Phys. Chem. Chem. Phys. 2013; 15, 10321.

[22] Nørskov, J.K.; Rossmeisl, J.; Logadottir, A.; Lindqvist, L.; Kitchin, J.R.; Bligaard, T.; Jónsson, H. Origin of the Overpotential for Oxygen Reduction at a Fuel-Cell Cathode. J. Phys. Chem. B 2004; 108, 17886-17892.

[23] Trasatti, S.; Parsons, R. Interphases in systems of conducting phases (Recommendations 1985). Pure Appl. Chem. 1986; 58, 437.

[24] Lozovoi, A.Y.; Alavi, A.; Kohanoff, J.; Lynden-Bell, R.M. Ab initio simulation of charged slabs at constant chemical potential. J. Chem. Phys. 2001; 115, 1661-1669.

[25] Otani, M.; Sugino, O. First-principles calculations of charged surfaces and interfaces: A plane-wave nonrepeated slab approach. Phys. Rev. B 2006; 73, 115407.

[26] Jinnouchi, R.; Anderson, A.B. Electronic structure calculations of liquid-solid interfaces: Combination of density functional theory and modified Poisson-Boltzmann theory. Phys. Rev. B 2008; 77, 245417.

[27] Letchworth-Weaver, K.; Arias, T.A. Joint density functional theory of the electrode-electrolyte interface: Application to fixed electrode potentials, interfacial capacitances, and potentials of zero charge. Phys. Rev. B 2012; 86, 075140 .

[28] Mathew, K.; Sundararaman, R.; Letchworth-Weaver, K.; Arias, T.A.; Hennig, R.G. Implicit solvation model for density-functional study of nanocrystal surfaces and reaction pathways. J. Chem. Phys. 2014; $140,84106$.

[29] Sundararaman, R.; GoddardIII, W.A.; Arias, T.A. Grand canonical electronic density-functional theory: Algorithms and applications to electrochemistry. J. Chem. Phys. 2017; 146, 114104.

[30] Han, B.; Li, Z.; Wandlowski, T. Adsorption and self-assembly of aromatic carboxylic acids on $\mathrm{Au} /$ electrolyte interfaces. Anal. Bioanal.Chem. 2007; 388, 121-129.

[31] Lambert, D.K. Vibrational Stark effect of adsorbates at electrochemical interfaces. Electrochim. Acta 1996; 41, 623-630.

[32] Ma, C.; Harris, J.M. Surface-enhanced raman spectroscopy investigation of the potential-dependent acid-base chemistry of silver-immobilized 2-mercaptobenzoic acid. Langmuir 2011; 27, 3527-3533.

[33] Wells, M.; Dermody, D.L.; Yang, H.C.; Kim, T.; Crooks, R.M.; Ricco, A.J. Interactions between Organized, Surface-Confined Monolayers and Vapor-Phase Probe Molecules. 9. Structure/Reactivity Relationship between Three Surface-Confined Isomers of Mercaptobenzoic Acid and Vapor-Phase Decylamine. Langmuir 1996; 12, 1989-1996.

[34] Pensa, E.; Rubert, A.A.; Benitez, G.; Carro, P.; Orive, A.G.; Creus, A.H.; Salvarezza, R.C.; Vericat, C. Are 4-mercaptobenzoic acid self assembled monolayers on $\mathrm{Au}(111)$ a suitable system to test adatom models? J. Phys. Chem. C 2012; 116, 25765-25771. 
[35] González, M.C.R.; Orive, A.G.; Carro, P.; Salvarezza, R.C.; Creus, A.H. Structure and Electronic and Charge-Transfer Properties of Mercaptobenzoic Acid and Mercaptobenzoic Acid-Undecanethiol Mixed Monolayers on $\mathrm{Au}(111)$. J. Phys. Chem. C 2014; 118, 30013-30022.

[36] Nara, J.; Higai, S.; Morikawa, Y.; Ohno, T. Density functional theory investigation of benzenethiol adsorption on $\mathrm{Au}(111)$. J. Chem. Phys. 2004; 120, 6705-6711.

[37] Wilson, E.B. The Normal Modes and Frequencies of Vibration of the Regular Plane Hexagon Model of the Benzene Molecule. Phys. Rev. 1934; 45, 706-714.

[38] Smith, C.P.; White, H.S. Voltammetry of Molecular Films Containing Acid/Base Groups. Langmuir $1993 ; 9,1-3$.

[39] Fawcett, W.R.; Fedurco, M.; Kováčová, Z. Double Layer Effects at Molecular Films Containing Acid/Base Groups. Langmuir 1994; 10, 2403-2408. 